\title{
Backward bifurcation, equilibrium and stability phenomena in a three-stage extended BRSV epidemic model
}

\author{
David Greenhalgh and Martin Griffiths
}

Department of Statistics and Modelling Science, University of Strathclyde, Livingstone Tower, 26 Richmond Street, Glasgow G1 1XH, U.K.

\begin{abstract}
In this paper we consider the phenomenon of backward bifurcation in epidemic modelling illustrated by an extended model for Bovine Respiratory Syncytial Virus (BRSV) amongst cattle. In its simplest form, backward bifurcation in epidemic models usually implies the existence of two subcritical endemic equilibria for $R_{0}<1$, where $R_{0}$ is the basic reproductive number, and a unique supercritical endemic equilibrium for $R_{0}>1$. In our three-stage extended model we find that more complex bifurcation diagrams are possible.

The paper starts with a review of some of the previous work on backward bifurcation then describes our three-stage model. We give equilibrium and stability results, and also provide some biological motivation for the model being studied. It is shown that backward bifurcation can occur in the three-stage model for small $b$, where $b$ is the common per capita birth and death rate. We are able to classify the possible bifurcation diagrams. Some realistic numerical examples are discussed at the end of the paper, both for $b$ small and for larger values of $b$.
\end{abstract}

Keywords:- Backward bifurcation, equilibrium and stability analysis, basic reproduction ratio, simulation, three stage model, Bovine Respiratory Syncytial Virus.

\section{Introduction and literature review}

The dynamics of the spread of an infectious disease in a population of humans or animals can, in many instances, be satisfactorily modelled by a system of deterministic differential equations. In obtaining these equations it is usual to assume that the population can be partitioned into a set of distinct classes, each defining the state of an individual with respect to the disease. In the simplest scenario an individual is either susceptible to the disease or infected, and our population is split into just two classes. To make things more realistic we might include further classes to take account of the various stages in the progression of the disease.

For a particular set of model parameters, we may search for equilibrium solutions of our system of differential equations. A bifurcation diagram provides a picture of how these equilibrium solutions depend on the basic reproduction ratio $R_{0}$, where $R_{0}$ is defined as the expected number of secondary cases caused by a single infected case entering the disease-free population at equilibrium. There is generally a change in the qualitative behaviour of the system when $R_{0}=1$ in that the disease-free equilibrium (DFE) bifurcates into a branch representing the endemic equilibrium and a further branch of the DFE. This point on the diagram is termed the bifurcation point, and the curve emanating from it the bifurcation curve. For most simple 
epidemic models no endemic equilibria are present when $R_{0} \leq 1$. In these cases the bifurcation curve is such that as we travel along it from the bifurcation point, the level of infection increases as $R_{0}$ increases. This is known as forward bifurcation. However, in more complicated disease models the phenomenon of backward bifurcation has also come to light, whereby the initial direction of the bifurcation curve is such that as we move along it from the bifurcation point, $R_{0}$ decreases as the level of infection increases.

It would appear that the first accounts of the presence of backward bifurcation in an epidemic model were given in similar articles by Castillo-Chavez et al. (1989a) and (1989b), and Huang et al. (1992). The phrase 'backward bifurcation' was not actually used in any of these papers, although Figure 1 in the article by Huang et al. (1992) is a bifurcation diagram that clearly demonstrates this phenomenon.

The continuous nature of a bifurcation curve means that the presence of backward bifurcation generally indicates the existence of (at least) two subcritical endemic equilibria for $R^{*}<R_{0}<1$, where $R^{*}$ corresponds to the value of $R_{0}$ at which a vertical turning point on the bifurcation curve occurs. It has been found in some models exhibiting backward bifurcation that one of these subcritical endemic equilibria, generally the one corresponding to the higher level of infection, is locally asymptotically stable (LAS). Thus the presence of backward bifurcation certainly has implications for disease control since it is now possible for the disease to persist even when $R_{0}<1$. In other words, the classical requirement for the eradication of the disease is no longer satisfied. In order to ensure that the disease is eliminated from the population we would require that $R_{0}<R^{*}$. It is also worth noting here that the presence of backward bifurcation is not actually necessary for the existence of multiple endemic equilibria. Indeed, as we shall show, it is possible for both multiple supercritical and multiple subcritical endemic equilibria to exist, even when a bifurcation diagram displays forward bifurcation.

Over the last decade it has emerged that a wide range of epidemic models have the potential for exhibiting multiple equilibria and, in particular, backward bifurcation. We summarise here a number of important papers that are directly relevant to our work here.

Greenhalgh et al. (2000) studied a model for the spread of Bovine Respiratory Syncytial Virus (BRSV) in cattle. The authors note that, although many models for the spread of infectious diseases amongst animals assume that any infected animal will transmit the disease at the same rate as any other, there is evidence that this may not always be the case. If recovery from an initial infection offers only partial immunity then it may be possible for an animal to become lightly infected again, maybe even without any observable clinical symptoms of the disease. It seems possible, for some diseases at least, that such seropositive animals may still be able to pass on the infection, but at a lower rate than animals experiencing infection for the first-time. The population in this model is thus partitioned into four classes, consisting of susceptible individuals that have never experienced the disease before, susceptible individuals that have been infected at least once before but are now recovered, individuals experiencing infection for the first time, and, finally infected individuals that have experienced infection before (the seropositive class). A detailed analysis reveals that backward bifurcation can occur, and that the higher of the two resulting subcritical endemic equilibria is stable while the lower one is unstable. Several bifurcation diagrams are obtained by fixing some of the parameter values for 
BRSV taken from de Jong et al. (1996) while varying the others. From these diagrams it would appear that there is the potential for backward bifurcation to occur using realistic parameter values.

It should be noted that the decision to use a two-stage model was for the sake of simplicity. In order to incorporate the idea that successive exposure to infectious agents may cause decreased susceptibility and infectivity, this might be regarded as an oversimplification. However, whilst in reality we probably need an infinite number of stages, three stages will give a more realistic model than a two-stage one. Also it is more important to model the first two stages separately as most of the important biological differences will occur during the first two stages. Indeed, this is something that we shall pursue in this paper.

The paper by Hadeler and Castillo-Chavez (1995) considers the spread of a sexually transmitted disease in a population split into a sexually active and relatively small core group, and a weakly connected and largely inactive remainder, referred to as the non-core group. An example of a particular disease they had in mind was HIV/AIDS, with the population being homosexual males. The core group is the promiscuous element of the homosexual community while the non-core group is regarded as completely inactive sexually. The core group is further subdivided into susceptible, educated (or vaccinated) and infected individuals. It was found that in the extreme cases where either education leads to complete protection or where all recovered individuals proceed immediately to the educated class, there is no backward bifurcation. The authors noted that the sets of parameter values that did actually lead to the presence of backward bifurcation are characteristic of a highly infective disease and a poor education program so that some educated individuals do become infected, and recovered individuals have a high risk of going back to the susceptible class. Again here, this model might be made more realistic by incorporating various levels of education.

Dushoff (1996) incorporates ideas from immunology into an epidemiological model. The author presents a simple model with one susceptible class and two possible states of infection, in which it is assumed that the more exposure an individual has to the infection the more likely they are to acquire the severe state of infection. However, an intermediate state might be more realistic giving a three stage model. That the intensity of exposure can have an affect on the immunological outcome is given theoretical support via the models of Schweitzer and Anderson (1992). In their paper they suggest that in some cases a low level of exposure to an infectious organism may lead to a mild infection whilst a greater exposure may overwhelm the immune system and lead to a far greater subsequent transmission of the disease. Three stages may be more accurate here too.

Hadeler and van den Driessche (1997) consider an SIRS model for two social groups with different susceptibilities to the disease, referred to as 'normal' or 'educated'. A third intermediate class would be appropriate here also.

Greenhalgh et al. (2001) examine the impact of condom use on the sexual transmission of HIV and AIDS in a homosexual male population. To this end they initially consider a general age-structured model for which the frequency of condom use is represented by a continuous distribution. The age structure is collapsed by discretising the frequency of condom use into $N$ distinct levels. The case $N=2$ is considered firstly in its most general form and then specialised to the situation where all individuals in one of the two groups are completely safe in their sexual practices. Infected individuals in the high risk group can migrate to the zero risk group. 
However, we would obtain a more realistic approximation by looking at a model with $N=3$, incorporating a group with an intermediate level of condom use.

On examining the available literature, it becomes clear that the investigation of backward bifurcation in epidemic modelling is a relatively new research area, and that there are still many aspects of this interesting phenomenon warranting further study. A number of the papers reviewed stated that more complicated versions of the epidemic model being studied would probably also exhibit backward bifurcation. This would certainly seem plausible in general. Furthermore, other than a cursory comment and accompanying diagram in a paper by van den Driessche and Watmough (2000), it does not seem to have been mentioned anywhere that more complex models could possibly lead to bifurcation diagrams that are even more interesting than those already considered. For relatively complicated (multi-stage, for example) disease models the shape of the bifurcation diagram may allow subcritical endemic equilibria and multiple supercritical endemic equilibria to exist while still displaying forward bifurcation. The possibility for the existence of these more complex bifurcation diagrams in epidemic modelling is something that we wish to investigate here. Should they exist, their interesting geometry could have ramifications with regard to the dynamics of the models in the regions of the parameter space near $R_{0}=1$.

We consider here a disease model that is a natural extension, to three susceptible and three infectious classes, of the two-stage BRSV model studied by Greenhalgh et al. (2000). We have two good reasons for choosing this particular model:

(1) Its relative complexity will provide the potential for some of the more complex bifurcation diagrams alluded to above. While the two-stage model is interesting in its own right and does, under certain circumstances, exhibit backward bifurcation, it does not allow the possibility for two subcritical endemic equilibria to exist in the presence of forward bifurcation, nor does it allow the existence of multiple supercritical equilibria.

(2) As we have argued previously, a three-stage model for the spread of BRSV in cattle may actually be more realistic than the already-existing two-stage model.

We probe our three-stage model analytically in order to determine which of these more interesting bifurcation diagrams are possible, and then go on to pursue a more in-depth analysis, with the intention of establishing a set of criteria (i.e. sets of conditions on the parameter values) such that when they are satisfied these interesting phenomena are able to occur. We then classify the possible shapes of the bifurcation diagrams, along with the regions of the parameter space that give rise to them. We would, of course, also want to consider whether any of our criteria for multiple endemic equilibria to occur can be satisfied by a set of biologically meaningful parameter values.

\section{The model}

We define here a three-stage model for the spread of an infectious disease through a population. This is an extension of the two-stage model for BRSV discussed by Greenhalgh et al. (2000). The basic idea is that infection confers partial but not complete immunity on individuals. We assume that the disease is not fatal. There are 
three susceptible classes: those who have never previously been infected $S_{1}$, those who have been infected just once $S_{2}$, and those who have suffered at least two previous infections $S_{3}$. Similarly, $I_{1}$ denotes the number of first-time infected individuals, $I_{2}$ the number experiencing infection for the second-time, and $I_{3}$ the number of infected individuals for which this is at least their third period of infection. Thus, if $N$ is the total population size, we have that $S_{1}+S_{2}+S_{3}+I_{1}+I_{2}+I_{3}=N$.

For an individual in infectious class $i, i=1,2,3$, the infectious period is assumed to be exponentially distributed with parameter $\beta_{i}$. First-time susceptible individuals are infected by individuals in infectious class $i$ at rate $\alpha_{i} I_{i} / N, i=1,2,3$. Susceptible individuals that have already experienced the disease will have partial immunity and we model this using the parameters $\gamma_{1}$ and $\gamma_{2}$ which are respectively the factors by which the infectivity is reduced for second and third-time susceptible individuals. Thus, for example, $\gamma_{1} \alpha_{2} I_{2} / N$ gives the per capita rate at which secondtime susceptible individuals are infected by second-time infected individuals. We assume that $1 \geq \gamma_{1} \geq \gamma_{2}>0$.

We also assume that the population size remains constant. We say that $b$ is the per capita birth rate and also the per capita death rate for each of the six categories. We also assume that $\phi$ is the proportion of individuals that are vaccinated at birth, and that these individuals immediately enter the class of third-time susceptible individuals. We have that $0 \leq \phi \leq 1$.

By defining $s_{j}=S_{j} / N$ and $i_{j}=I_{j} / N, j=1,2,3$, the spread of the infection in the above model can be described by the following system of differential equations:

$$
\begin{aligned}
\frac{d s_{1}}{d t} & =b(1-\phi)-\left(\alpha_{1} i_{1}+\alpha_{2} i_{2}+\alpha_{3} i_{3}\right) s_{1}-b s_{1}, \\
\frac{d i_{1}}{d t} & =\left(\alpha_{1} i_{1}+\alpha_{2} i_{2}+\alpha_{3} i_{3}\right) s_{1}-\left(b+\beta_{1}\right) i_{1}, \\
\frac{d s_{2}}{d t} & =\beta_{1} i_{1}-\gamma_{1}\left(\alpha_{1} i_{1}+\alpha_{2} i_{2}+\alpha_{3} i_{3}\right) s_{2}-b s_{2}, \\
\frac{d i_{2}}{d t} & =\gamma_{1}\left(\alpha_{1} i_{1}+\alpha_{2} i_{2}+\alpha_{3} i_{3}\right) s_{2}-\left(b+\beta_{2}\right) i_{2}, \\
\frac{d s_{3}}{d t} & =b \phi+\beta_{2} i_{2}+\beta_{3} i_{3}-\gamma_{2}\left(\alpha_{1} i_{1}+\alpha_{2} i_{2}+\alpha_{3} i_{3}\right) s_{3}-b s_{3} \\
\frac{d i_{3}}{d t} & =\gamma_{2}\left(\alpha_{1} i_{1}+\alpha_{2} i_{2}+\alpha_{3} i_{3}\right) s_{3}-\left(b+\beta_{3}\right) i_{3},
\end{aligned}
$$

and

where $s_{1}(t)+s_{2}(t)+s_{3}(t)+i_{1}(t)+i_{2}(t)+i_{3}(t)=1$ for all $t \geq 0$, and $s_{i}(0), i_{j}(0) \geq 0$ for $i, j=1,2,3$. We also assume that all parameter values, apart from $\phi$ are strictly positive.

Simple models assume that all animals are equally susceptible or infectious, but the reality is that is that successive exposure to infectious agents may cause decreased susceptibility and infectivity. For the sake of simplicity Greenhalgh et al. (2000) used a two-stage model for BRSV in cattle, but whilst in reality we probably need an infinite number of stages, three stages gives a more realistic model than does two. Also, it is important to model the first two stages separately as most of the 
important changes will be covered by them. The term $\beta_{3} i_{3}$ in the equation for $s_{3}$ is justified as this represents the return of individuals into the susceptible population after having recovered from their third (and any subsequent) infectious period.

Ideally even more stages would be needed (indeed, this idea is examined by Griffiths (2007)) but this greatly increases the difficulty in classifying the possible bifurcation diagrams. Also, given that there are a large number of two-stage models which exhibit backward bifurcation, all of which have qualitatively similar bifurcation diagrams, and that most of these models are similarly approximations to a more general situation where there are an infinite number of groups, it is of generic interest to examine how this model extends to three stages. This may have implications for realistic extensions of other two-stage models, such as the one studied by Hadeler and Castillo-Chavez (1995).

\subsection{Stability results for the disease-free equilibrium}

We note that there is a unique disease-free equilibrium (DFE) $\left(s_{1}, i_{1}, s_{2}, i_{2}, s_{3}, i_{3}\right)=(1-\phi, 0,0,0, \phi, 0)$. We first deduce the value of the basic reproduction ratio $R_{0}$ for this model. Note that $R_{0}$ is the dominant eigenvalue of the next generation matrix at the DFE (see Diekmann et al. (1991)).

If there are $k$ classes of infected individuals, the next generation matrix $\mathbf{m}$ is defined to be the $k \times k$ matrix such that the entry $m_{i j}$ is the expected number of infections caused in the $j$ th susceptible class by the introduction into the population at the DFE of a single individual from the $i$ th infected class. It is straightforward to show that

$$
\mathbf{m}=\left(\begin{array}{ccc}
\frac{\alpha_{1}(1-\phi)}{b+\beta_{1}} & 0 & \frac{\gamma_{2} \alpha_{1} \phi}{b+\beta_{1}} \\
\frac{\alpha_{2}(1-\phi)}{b+\beta_{2}} & 0 & \frac{\gamma_{2} \alpha_{2} \phi}{b+\beta_{2}} \\
\frac{\alpha_{3}(1-\phi)}{b+\beta_{3}} & 0 & \frac{\gamma_{2} \alpha_{3} \phi}{b+\beta_{3}}
\end{array}\right)
$$

and hence $R_{0}=\frac{\alpha_{1}(1-\phi)}{b+\beta_{1}}+\frac{\gamma_{2} \alpha_{3} \phi}{b+\beta_{3}}$. Using the results in Diekmann et al. (1991) we know that the DFE is LAS when $R_{0}<1$ and unstable when $R_{0}>1$. We are also able to give a global stability result for the DFE:

Theorem 2.1.1 The DFE, $\left(s_{1}, i_{1}, s_{2}, i_{2}, s_{3}, i_{3}\right)=(1-\phi, 0,0,0, \phi, 0)$, is globally asymptotically stable (GAS) if $1>(1-\phi) R_{01}+R_{02}+R_{03}$, where

$$
R_{01}=\frac{\alpha_{1}}{b+\beta_{1}}, R_{02}=\frac{\gamma_{1} \alpha_{2}}{b+\beta_{2}} \text { and } R_{03}=\frac{\gamma_{2} \alpha_{3}}{b+\beta_{3}}
$$

Proof Because of the similarity of the equation for $d s_{1} / d t$ to the one for the twostage model (Greenhalgh et al., 2000) we can use a similar argument to obtain the 
result $s_{1}^{\infty}=\limsup _{t \rightarrow \infty} s_{1}(T) \leq 1-\phi$. Then, for any $\varepsilon>0$ there exists some $t_{0}$ such that $s_{1} \leq 1-\phi+\varepsilon$ for all $t \geq t_{0}$. Pick $\varepsilon$ small enough so that $1>(1-\phi+\varepsilon) R_{01}+R_{02}+R_{03}$. We now have, for $t \geq t_{0}$, using $s_{1} \leq 1-\phi+\varepsilon$,

$$
\frac{d i_{1}}{d t} \leq i_{1}\left\{\alpha_{1}(1-\phi+\varepsilon)-\left(b+\beta_{1}\right)\right\}+i_{2}\left\{\alpha_{2}(1-\phi+\varepsilon)\right\}+i_{3}\left\{\alpha_{3}(1-\phi+\varepsilon)\right\} .
$$

Also, since $s_{2} \leq 1$ and $s_{3} \leq 1$, we have that

$$
\frac{d i_{2}}{d t} \leq i_{1}\left\{\gamma_{1} \alpha_{1}\right\}+i_{2}\left\{\gamma_{1} \alpha_{2}-\left(b+\beta_{2}\right)\right\}+i_{3}\left\{\gamma_{1} \alpha_{3}\right\}
$$

and

$$
\frac{d i_{3}}{d t} \leq i_{1}\left\{\gamma_{2} \alpha_{1}\right\}+i_{2}\left\{\gamma_{2} \alpha_{2}\right\}+i_{3}\left\{\gamma_{2} \alpha_{3}-\left(b+\beta_{3}\right)\right\} \text {. }
$$

Thus $d \mathbf{i} / d t \leq \mathbf{Q i}$ where $\mathbf{i}=\left(i_{1}, i_{2}, i_{3}\right)^{\mathrm{T}}$ and

$$
\mathbf{Q}=\left(\begin{array}{ccc}
\alpha_{1}(1-\phi+\varepsilon)-\left(b+\beta_{1}\right) & \alpha_{2}(1-\phi+\varepsilon) & \alpha_{3}(1-\phi+\varepsilon) \\
\gamma_{1} \alpha_{1} & \gamma_{1} \alpha_{2}-\left(b+\beta_{2}\right) & \gamma_{1} \alpha_{3} \\
\gamma_{2} \alpha_{1} & \gamma_{2} \alpha_{2} & \gamma_{2} \alpha_{3}-\left(b+\beta_{3}\right)
\end{array}\right) .
$$

$\mathbf{Q}+M \mathbf{I}$ is a matrix with strictly positive entries so long as $M$ is a large enough real number. Thus, by the Perron-Frobenius Theorem (see Varga (1962)), Q+MI has a simple eigenvalue, $\lambda$ say, equal to its spectral radius, and the corresponding left eigenvector $\mathbf{e}$ has strictly positive entries. Then $\mathbf{e}$ is also an eigenvector of $\mathbf{Q}$ with corresponding eigenvalue $\lambda-M$. Note that the eigenvalue corresponding to the spectral radius is the dominant eigenvalue, and that the spectral radius, and hence $\lambda$, is always a positive real number. If we let $\omega_{1}=\lambda-M, \omega_{2}$ and $\omega_{3}$ be the eigenvalues of $\mathbf{Q}$ then the eigenvalues of $\mathbf{Q}+M \mathbf{I}$ are $\omega_{1}+M, \omega_{2}+M$ and $\omega_{3}+M$. Thus $\omega_{1}$ is the dominant eigenvalue of $\mathbf{Q}$.

The characteristic equation of $\mathbf{Q}$ is $\operatorname{det}[\mathbf{Q}-\omega \mathbf{I}]=0$, and this is given by $\omega^{3}+A \omega^{2}+B \omega+C=0$, where

$$
\begin{aligned}
A= & \left(b+\beta_{1}\right)+\left(b+\beta_{2}\right)+\left(b+\beta_{3}\right)-\alpha_{1}(1-\phi+\varepsilon)-\gamma_{1} \alpha_{2}-\gamma_{2} \alpha_{3}, \\
B= & \left(b+\beta_{1}\right)\left(b+\beta_{2}\right)+\left(b+\beta_{1}\right)\left(b+\beta_{3}\right)+\left(b+\beta_{2}\right)\left(b+\beta_{3}\right)-\alpha_{1}(1+\phi+\varepsilon)\left\{\left(b+\beta_{2}\right)+\left(b+\beta_{3}\right)\right\} \\
& -\gamma_{1} \alpha_{2}\left\{\left(b+\beta_{1}\right)+\left(b+\beta_{3}\right)\right\}-\gamma_{2} \alpha_{3}\left\{\left(b+\beta_{1}\right)+\left(b+\beta_{2}\right)\right\},
\end{aligned}
$$

and

$$
\begin{aligned}
C= & \left(b+\beta_{1}\right)\left(b+\beta_{2}\right)\left(b+\beta_{3}\right)-\alpha_{1}(1-\phi+\varepsilon)\left(b+\beta_{2}\right)\left(b+\beta_{3}\right)-\gamma_{1} \alpha_{2}\left(b+\beta_{1}\right)\left(b+\beta_{3}\right) \\
& -\gamma_{2} \alpha_{3}\left(b+\beta_{1}\right)\left(b+\beta_{2}\right) .
\end{aligned}
$$

For all the roots $\omega_{1}, \omega_{2}$ and $\omega_{3}$ of $\operatorname{det}[\mathbf{Q}-\omega \mathbf{I}]=0$ to have negative real parts then, by the Routh-Hurwitz criteria, we need $A$ and $C$ positive with $A B>C$. Note that $C>0$ if, and only if $1>(1-\phi+\varepsilon) R_{01}+R_{02}+R_{03}$. Next, as $1>(1-\phi+\varepsilon) R_{01}+R_{02}+R_{03} \quad$ then $b+\beta_{1}>\alpha_{1}(1-\phi+\varepsilon), \quad b+\beta_{2}>\gamma_{1} \alpha_{2} \quad$ and $b+\beta_{3}>\gamma_{2} \alpha_{3}$, implying that $A>0$. 
It remains, therefore, to check that $A B>C$. This is true as a consequence of Lemma 2.1.2 below. Hence $\omega_{1}$ is a negative real number. Since e has strictly positive entries then the fact that $d \mathbf{i} / d t \leq \mathbf{Q i}$ tells us that $d(\mathbf{e . i}) / d t \leq(\mathbf{e Q}) . \mathbf{i}=\omega_{1} \mathbf{e . i}$. On integrating we see that $\mathbf{e . i} \rightarrow 0$ as $t \rightarrow \infty$. As e is strictly positive, we see that $\mathbf{i} \rightarrow \mathbf{0}$ as $t \rightarrow \infty$. Then, as in Greenhalgh et al. (2000), it can be shown that $s_{1} \rightarrow 1-\phi$ as $t \rightarrow \infty$.

Since $d s_{2} / d t=\beta_{1} i_{1}-\gamma_{1}\left(\alpha_{1} i_{1}+\alpha_{2} i_{2}+\alpha_{3} i_{3}\right) s_{2}-b s_{2}$, then the fact that $\mathbf{i} \rightarrow \mathbf{0}$ as $t \rightarrow \infty$ means that $s_{2} \rightarrow 0$ as $t \rightarrow \infty$. Finally, the above results imply that $s_{3} \rightarrow \phi$ as $t \rightarrow \infty$, showing that the DFE $\left(s_{1}, i_{1}, s_{2}, i_{2}, s_{3}, i_{3}\right)=(1-\phi, 0,0,0, \phi, 0)$ is GAS if $1>(1-\phi+\varepsilon) R_{01}+R_{02}+R_{03}$.

Lemma 2.1.2 If $a, b, c, d, e$ and $f$ are positive real numbers such that $\frac{d}{a}+\frac{e}{b}+\frac{f}{c}<1$ then

$$
\begin{array}{r}
(a+b+c-d-e-f) a b+b c+a c-d(b+c)-e(a+c)-f(a+b) \\
>(a b c-d b c-e a c-f a b) .
\end{array}
$$

Proof See Appendix.

\section{The existence of endemic equilibria}

Letting $\left(s_{1}^{*}, i_{1}^{*}, s_{2}^{*}, i_{2}^{*}, s_{3}^{*}, i_{3}^{*}\right)$ denote an endemic equilibrium solution then, with $I=\alpha_{1} i_{1}^{*}+\alpha_{2} i_{2}^{*}+\alpha_{3} i_{3}^{*}$, we obtain, from the differential equations:

$$
\begin{aligned}
& s_{1}^{*}=\frac{b(1-\phi)}{I+b}=\frac{\left(b+\beta_{1}\right) i_{1}^{*}}{I}, \\
& s_{2}^{*}=\frac{\beta_{1} i_{1}^{*}}{\gamma_{1} I+b}=\frac{\left(b+\beta_{2}\right) i_{2}^{*}}{\gamma_{1} I},
\end{aligned}
$$

and $\quad s_{3}^{*}=\frac{b \phi+\beta_{2} i_{2}^{*}+\beta_{3} i_{3}^{*}}{\gamma_{2} I+b}=\frac{\left(b+\beta_{3}\right) i_{3}^{*}}{\gamma_{2} I}$.

From these we have

and

$$
\begin{aligned}
& i_{1}^{*}=\frac{b(1-\phi) I}{\left(b+\beta_{1}\right)(I+b)}, \\
& i_{2}^{*}=\frac{\gamma_{1} \beta_{1} b(1-\phi) I^{2}}{\left(b+\beta_{1}\right)\left(b+\beta_{2}\right)(I+b)\left(\gamma_{1} I+b\right)}, \\
& i_{3}^{*}=\frac{\gamma_{2} \phi I}{\left(\gamma_{2} I+b+\beta_{3}\right)}+\frac{\gamma_{1} \gamma_{2} \beta_{1} \beta_{2}(1-\phi) I^{3}}{\left(b+\beta_{1}\right)\left(b+\beta_{2}\right)(I+b)\left(\gamma_{1} I+b\right)\left(\gamma_{2} I+b+\beta_{3}\right)} .
\end{aligned}
$$

Now, on using $I=\alpha_{1} i_{1}^{*}+\alpha_{2} i_{2}^{*}+\alpha_{3} i_{3}^{*}$, we obtain the following equation in $I$ (that we shall later transform into a cubic in $I$ ): 


$$
\begin{aligned}
& \left(b+\beta_{1}\right)\left(b+\beta_{2}\right)= \\
& \frac{\alpha_{1} b(1-\phi)\left(b+\beta_{2}\right)}{I+b}+\frac{\alpha_{2} \beta_{1} b(1-\phi) I}{(I+b)\left(I+\frac{b}{\gamma_{1}}\right)}+\frac{\alpha_{3} \beta_{1} \beta_{2}(1-\phi) I^{2}}{(I+b)\left(I+\frac{b}{\gamma_{1}}\right)\left(I+\frac{b+\beta_{3}}{\gamma_{2}}\right)}+\frac{\alpha_{3} \phi\left(b+\beta_{1}\right)\left(b+\beta_{2}\right)}{I+\frac{b+\beta_{3}}{\gamma_{2}}} .
\end{aligned}
$$

It is straightforward to show that there is a one-to-one correspondence between the endemic equilibria of the model and the positive real roots of equation (3.1) for $I$. We note here that since $0 \leq i_{1}^{*}+i_{2}^{*}+i_{3}^{*} \leq 1$ we have that $0 \leq I \leq \max \alpha_{1}, \alpha_{2}, \alpha_{3}$.

\subsection{The existence of backward bifurcation and multiple endemic equilibria}

Let us firstly deal with the case for which all individuals are vaccinated at birth. In this case we have $\phi=1$ and our equation in $I$ simplifies considerably to

$$
\frac{\alpha_{3}\left(b+\beta_{1}\right)\left(b+\beta_{2}\right)}{I+\frac{b+\beta_{3}}{\gamma_{2}}}=\left(b+\beta_{1}\right)\left(b+\beta_{2}\right) \text {. }
$$

This has the unique solution $I=\alpha_{3}-\left(b+\beta_{3}\right) / \gamma_{2}$. For this to be an endemic equilibrium, we require $\alpha_{3}-\left(b+\beta_{3}\right) / \gamma_{2}>0$, which is equivalent to the condition $R_{0}>1$. We see, therefore, the usual forward bifurcation occurring here. There is a unique endemic equilibrium if, and only if, $R_{0}>1$.

Having dealt with the special case $\phi=1$ we shall, from now on, assume that $0 \leq \phi<1$. The equation in $I$ can be re-arranged to give

with $A=\left(b+\beta_{1}\right)\left(b+\beta_{2}\right)$,

$$
A I^{3}+B I^{2}+C I+D=0
$$

$$
\begin{gathered}
B=\left(b+\beta_{1}\right)\left(b+\beta_{2}\right) b+\frac{b}{\gamma_{1}}+\frac{b+\beta_{3}}{\gamma_{2}}-\alpha_{1} b(1-\phi)\left(b+\beta_{2}\right)-\alpha_{2} \beta_{1} b(1-\phi) \\
-\alpha_{3} \beta_{1} \beta_{2}(1-\phi)-\alpha_{3} \phi\left(b+\beta_{1}\right)\left(b+\beta_{2}\right), \\
C=b\left(b+\beta_{1}\right)\left(b+\beta_{2}\right) \frac{b}{\gamma_{1}}+\frac{b+\beta_{3}}{\gamma_{2}}+\frac{b+\beta_{3}}{\gamma_{1} \gamma_{2}}-\alpha_{1} b(1-\phi)\left(b+\beta_{2}\right) \frac{b}{\gamma_{1}}+\frac{b+\beta_{3}}{\gamma_{2}} \\
-\alpha_{2} \beta_{1} b 1-\phi \frac{b+\beta_{3}}{\gamma_{2}}-\alpha_{3} b \phi\left(b+\beta_{1}\right)\left(b+\beta_{2}\right) 1+\frac{1}{\gamma_{1}},
\end{gathered}
$$

and $D=\frac{b^{2}}{\gamma_{1} \gamma_{2}}\left(b+\beta_{1}\right)\left(b+\beta_{2}\right)\left(b+\beta_{3}\right)\left\{1-R_{0}\right\}$.

From the above we see that $R_{0}<1$ if, and only if, $D>0$. Thus, by considering the shape of the graph $y=A I^{3}+B I^{2}+C I+D$ (and noting that $A>0$ ), we have that there will either be zero or two subcritical endemic equilibria in this case.

In order to show that two subcritical endemic equilibria are actually possible (accompanied by backward bifurcation), we firstly obtain the following result:

Lemma 3.1 Consider the function $f(x, \lambda)=a x^{3}+b x^{2}+c x+d$ where $a>0$ is fixed but for which the coefficients $b, c$ and $d$ are each continuous functions of some parameter $\lambda$ where, in particular, $d$ is a strictly decreasing function of $\lambda$. Suppose 
that, for some $\lambda=\lambda_{d}>0$, we have $c\left(\lambda_{d}\right)<0$ and $d\left(\lambda_{d}\right)=0$. Then there exists some $\lambda=\lambda_{1}>0$ (where $\left.d\left(\lambda_{1}\right)>0\right)$ such that $f(x, \lambda)=0$ has two positive real roots for all $d$ in $0, d\left(\lambda_{1}\right)$.

Proof See Appendix.

Theorem 3.2 It is possible for backward bifurcation to occur when $0 \leq \phi<1$.

Proof We shall introduce the bifurcation coordinate á $=\left(\alpha_{1}, \alpha_{2}, \alpha_{3}\right)$, and assume that the remaining parameters are set at some fixed positive values. Now we choose any value for $\alpha_{3}, \overline{\alpha_{3}}$ say, such that $\frac{b+\beta_{1}}{1-\phi}\left(1-\frac{\gamma_{2} \overline{\alpha_{3}} \phi}{b+\beta_{3}}\right)>0$. We will consider the 3dimensional parameter space comprising of all elements $\mathbf{a}=\left(\alpha_{1}, \alpha_{2}, \alpha_{3}\right)$ in the positive octant. If we choose $\alpha_{1}$ as our bifurcation parameter, the expression on the left hand side of the above inequality gives $\alpha_{1}\left(R_{0}=1\right)$, the value of $\alpha_{1}$ corresponding to $R_{0}=1$. Note that we have a free choice for $\alpha_{2}$.

We have

$$
\begin{array}{r}
C=b\left\{\left(b+\beta_{1}\right)\left(b+\beta_{2}\right)\left\{\left(\frac{b}{\gamma_{1}}+\frac{b+\beta_{3}}{\gamma_{2}}\right)\left(1-\frac{\alpha_{1}(1-\phi)}{b+\beta_{1}}-\frac{\gamma_{2} \alpha_{3} \phi}{b+\beta_{3}}\right)+\frac{\alpha_{3} \phi}{\gamma_{1}}\left(\frac{b \gamma_{2}}{b+\beta_{3}}-1\right)\right\}\right. \\
\left.+\frac{b+\beta_{3}}{\gamma_{1} \gamma_{2}}\left(b+\beta_{1}\right)\left(b+\beta_{2}\right)-\gamma_{1} \alpha_{2} \beta_{1}(1-\phi)\right\} .
\end{array}
$$

In particular $C\left(R_{0}=1\right)=$

$$
\frac{b}{\gamma_{1}}\left\{\alpha_{3} \phi\left(b+\beta_{1}\right)\left(b+\beta_{2}\right)\left(\frac{b \gamma_{2}}{b+\beta_{3}}-1\right)+\frac{b+\beta_{3}}{\gamma_{2}}\left(b+\beta_{1}\right)\left(b+\beta_{2}\right)-\gamma_{1} \alpha_{2} \beta_{1}(1-\phi)\right\} .
$$

We see that it is possible to choose some positive value of $\alpha_{2}, \bar{\alpha}_{2}$ say, such that $C\left(R_{0}=1\right)<0$. We now consider the behaviour of the graph $y=A I^{3}+B I^{2}+C I+D$, with á $=\left(\alpha_{1}, \bar{\alpha}_{2}, \bar{\alpha}_{3}\right)$, as we decrease $\alpha_{1}$ from the value $\alpha_{1}\left(R_{0}=1\right)$, noting that $B, C$ and $D$ are each continuous functions of $\alpha_{1}$, $D\left(R_{0}=1\right)=0$, and $D$ decreases with $\alpha_{1}$. Lemma 3.1 tells us that there exists some $\hat{\alpha}_{1}$ satisfying $0<\hat{\alpha}_{1}<\alpha_{1}\left(R_{0}=1\right)$ such that our model possesses two subcritical endemic equilibria for á $=\left(\alpha_{1}, \bar{\alpha}_{2}, \bar{\alpha}_{3}\right)$ whenever $\hat{\alpha}_{1}<\alpha_{1}<\alpha_{1}\left(R_{0}=1\right)$. In other words, we have shown that it is possible for there to exist two endemic equilibria when $R_{0}<1$.

We have already noted that the existence of two subcritical endemic equilibria does not necessarily imply the presence of backward bifurcation. However, so long as $C\left(R_{0}=1\right)<0$, we will have backward bifurcation here. This may be explained as follows: The graph of $y=A I^{3}+B I^{2}+C I+D$ passes through the origin when 
$R_{0}=1$, and the gradient here is given by $C\left(R_{0}=1\right)$. Thus when $C\left(R_{0}=1\right)<0$ the graph has a negative gradient at the origin. We also know that $D$ is a decreasing function of $\alpha_{1}$. Therefore, by continuity we see that for some $\varepsilon>0$, there is only one endemic equilibrium for $\alpha_{1} \in \alpha_{1}\left(R_{0}=1\right), \alpha_{1}\left(R_{0}=1\right)+\varepsilon$ but two endemic equilibria for $\alpha_{1} \in \alpha_{1}\left(R_{0}=1\right)-\varepsilon, \alpha_{1}\left(R_{0}=1\right)$. Hence backward bifurcation is present when $C\left(R_{0}=1\right)<0$. In fact it is clear that there exists some non-empty connected subspace, $S$ say, of $\Re^{3}$ such that backward bifurcation occurs for all $\left(\alpha_{1}, \alpha_{2}, \alpha_{3}\right) \in S$.

We note that the conditions $C\left(R_{0}=1\right)<0$ and $C\left(R_{0}=1\right)>0$ tell us backward and forward bifurcation will occur respectively. The latter, however, does not preclude the existence of subcritical endemic equilibria.

\section{Determination of regions with different numbers of endemic equilibria for the special case $b$ small}

We seek here regions of the parameter space $P$ for which multiple endemic equilibria can occur. We have already shown that this phenomenon does not occur when $\phi=1$, and we assume here that $0 \leq \phi<1$. Our equilibrium equation 3.2 may be written as $I^{3}+\hat{B} I^{2}+\hat{C} I+\hat{D}=0$, where

$$
\begin{gathered}
\hat{B}=b+\frac{b}{\gamma_{1}}+\frac{b+\beta_{3}}{\gamma_{2}}-\frac{\alpha_{1} b(1-\phi)}{b+\beta_{1}}-\frac{\alpha_{2} \beta_{1} b(1-\phi)}{\left(b+\beta_{1}\right)\left(b+\beta_{2}\right)}-\frac{\alpha_{3} \beta_{1} \beta_{2}(1-\phi)}{\left(b+\beta_{1}\right)\left(b+\beta_{2}\right)}-\alpha_{3} \phi, \\
\hat{C}=b\left\{\frac{b}{\gamma_{1}}+\frac{b+\beta_{3}}{\gamma_{2}}+\frac{b+\beta_{3}}{\gamma_{1} \gamma_{2}}-\frac{\alpha_{1} b(1-\phi)}{\gamma_{1}\left(b+\beta_{1}\right)}-\frac{\alpha_{1}\left(b+\beta_{3}\right)(1-\phi)}{\gamma_{2}\left(b+\beta_{1}\right)}\right. \\
\left.-\frac{\alpha_{2} \beta_{1}\left(b+\beta_{3}\right)(1-\phi)}{\gamma_{2}\left(b+\beta_{1}\right)\left(b+\beta_{2}\right)}-\alpha_{3} \phi\left(1+\frac{1}{\gamma_{1}}\right)\right\}
\end{gathered}
$$

and $\hat{D}=\frac{b^{2}}{\gamma_{1} \gamma_{2}}\left(b+\beta_{3}\right)\left\{1-\frac{\alpha_{1}(1-\phi)}{b+\beta_{1}}-\frac{\gamma_{2} \alpha_{3} \phi}{b+\beta_{3}}\right\}=\frac{b^{2}}{\gamma_{1} \gamma_{2}}\left(b+\beta_{3}\right) 1-R_{0}$.

In practice $b$ tends to be numerically very small compared to the other parameters as the disease spreads over a much shorter timescale than the lifetime of the animals.

For notational convenience we now define the following:

$$
R_{1}=\frac{\alpha_{1}}{\beta_{1}}, R_{2}=\frac{\gamma_{1} \alpha_{2}}{\beta_{2}} \text { and } R_{3}=\frac{\gamma_{2} \alpha_{3}}{\beta_{3}}
$$

These expressions are independent of one another. Note that as $b$ is small $R_{i} \approx R_{0 i}$ and $R_{i}=\lim _{b \rightarrow 0} R_{0 i}$ for $i=1,2,3 . R_{01}, R_{02}$ and $R_{03}$ each have a biological interpretation with regard to our disease model. $R_{01}$ is the basic reproduction ratio for an SIS model in which the per capita contact rate of susceptible animals is $\alpha_{1}$ and the average infectious period is $\beta_{1}^{-1}$. Similarly, $R_{02}$ is the basic reproduction ratio for an SIS model in which the per capita contact rate of susceptible animals is $\alpha_{2}$, but for which a contact between a susceptible and an infected animal results in an infection with 
probability $\gamma_{1} \cdot R_{03}$ has a corresponding interpretation. It should also be noted that as bifurcation parameters, $R_{1}$ and $\alpha_{1}$ can be regarded as essentially equivalent.

Using the above definitions and assuming that $b$ is small we have that

$$
\begin{aligned}
& \hat{B}=\frac{\beta_{3}}{\gamma_{2}}\left(1-R_{3}\right)+O(b), \\
& \hat{C}=\frac{b \beta_{3}}{\gamma_{1} \gamma_{2}}\left(1+\gamma_{1}\right)\left(1-\phi R_{3}\right)-(1-\phi)\left(\gamma_{1} R_{1}+R_{2}\right)+O\left(b^{2}\right)
\end{aligned}
$$

and

$$
\hat{D}=\frac{b^{2} \beta_{3}}{\gamma_{1} \gamma_{2}} 1-(1-\phi) R_{1}-\phi R_{3}+O\left(b^{3}\right) \text {. }
$$

Now let $F(I)=I^{3}+\hat{B} I^{2}+\hat{C} I+\hat{D}$ so that $F^{\prime}(I)=3 I^{2}+2 \hat{B} I+\hat{C}$. We already know that there is a one-to-one correspondence between the positive roots of $F(I)=0$ and the endemic equilibria, and we next determine the regions in the parameter space $P$ for which there are different numbers of endemic equilibria.

\subsection{Subcritical endemic equilibria}

We start by considering the case $R_{0}<1$. In order to state the intervals of interest, we now define certain 'critical' values of $R_{1}$ :

$R_{1}^{a}=\frac{1-\phi R_{3}}{1-\phi}$ arising from the requirement that $R_{0}<1$,

$R_{1}^{b}=\frac{1}{\gamma_{1}}\left(1+\gamma_{1}\right) R_{1}^{a}-R_{2}$ from the upper limit on $R_{3}$ in (A.3),

$R_{1}^{c}=R_{2}$ from the lower and upper limits on $R_{2}$ in (A.3) and (A.4), respectively, and

$R_{1}^{d}=\frac{1}{\gamma_{1}} 1+\gamma_{1}-R_{2}$ from the upper limit on $R_{2}$ in (A.3).

Finally, condition on $R_{3}<1$ and,

$$
\frac{1}{1-\phi}\left(1+\gamma_{1}\right)\left(1-\phi R_{3}\right)+2 \sqrt{\gamma_{1}\left(1-R_{3}\right) 1-\phi R_{3}}<R_{2}<R_{1}^{a}
$$

Let $R_{1}^{e}$ be the unique positive root of

$$
R_{2}=\frac{1}{1-\phi}\left(1+\gamma_{1}\right)\left(1-\phi R_{3}\right)+2 \sqrt{\gamma_{1}\left(1-R_{3}\right) 1-(1-\phi) R_{1}-\phi R_{3}}-\gamma_{1} R_{1}
$$

in $\left(0, R_{1}^{a}\right] . \quad R_{1}^{e}$ arises from the lower limit on $R_{2}$ in (A.8). 
Theorem 4.1 For sufficiently small $b$ :

(1) If $R_{3}>1 / \phi$ then there are no subcritical endemic equilibria.

(2) If $1<R_{3}<1 / \phi$ then there are two subcritical endemic equilibria for all $R_{1} \in\left(0, R_{1}^{a}\right)$, and two subcritical endemic equilibria for all $R_{1} \in\left(0, R_{1}^{a}\right]$ except possibly when $R_{2}=R_{1}^{a}$.

(3) If $R_{3} \leq 1$ and $R_{2} \leq R_{1}^{a}$ then there are no subcritical endemic equilibria for all $R_{1} \in\left(0, R_{1}^{a}\right)$, and no subcritical endemic equilibria for all $R_{1} \in\left(0, R_{1}^{a}\right]$ except possibly when $R_{2}=R_{1}^{a}$.

(4) If $R_{3} \leq 1$ and $R_{1}^{a}<R_{2}<\frac{1}{1-\phi}\left(1+\gamma_{1}\right)\left(1-\phi R_{3}\right)+2 \sqrt{\gamma_{1}\left(1-R_{3}\right) 1-\phi R_{3}}$ then there are two subcritical endemic equilibria for all $R_{1} \in\left(R_{1}^{e}, R_{1}^{a}\right]$ and no subcritical endemic equilibria for all $R_{1} \in\left(0, R_{1}^{e}\right)$.

(5) If $R_{3} \leq 1$ and $R_{2} \geq \frac{1}{1-\phi}\left(1+\gamma_{1}\right)\left(1-\phi R_{3}\right)+2 \sqrt{\gamma_{1}\left(1-R_{3}\right) 1-\phi R_{3}}$ then there are two subcritical endemic equilibria for all $R_{1} \in\left(0, R_{1}^{a}\right]$.

Proof See Appendix.

In the conclusions section we consider the possibility of interpreting biologically the conditions given above. They cover all possible regions of the parameter space $P$, with the exception of a finite number of special case equalities, defining curves and surfaces in $P$. The analysis is not sufficient to tell us whether or not there exist two subcritical endemic equilibria in these cases. We would need to consider higher order terms in $b$.

\subsection{Multiple supercritical endemic equilibria}

Let us go on to consider the case $R_{0}>1$. We define

$$
R_{1}^{t}=\frac{1}{1-\phi}\left(1-\phi R_{3}+\frac{1}{\gamma_{1}}(2-\phi) R_{3}-1-2 \sqrt{(1-\phi) R_{3}\left(R_{3}-1\right)}\right) .
$$

Theorem 4.2 For $b$ sufficiently small:

(1) If $R_{3}>1 / \phi$ then there is only one supercritical endemic equilibrium for all $R_{1} \in\left(R_{1}^{a}, \infty\right)$.

(2) If $1<R_{3}<1 / \phi$ and $R_{2}<R_{1}^{a}$ then there are three supercritical endemic equilibria for all $R_{1} \in\left(R_{1}^{a}, R_{1}^{*}\right)$ and only one supercritical endemic equilibrium for all $R_{1} \in\left(R_{1}^{*}, \infty\right)$, where $R_{1}^{*}$ is the unique root in $\left(R_{1}^{a}, R_{1}^{t}\right)$ of the equation

$$
R_{2}=\frac{1}{1-\phi}\left(1+\gamma_{1}\right)\left(1-\phi R_{3}\right)-2 \sqrt{\gamma_{1}\left(R_{3}-1\right)(1-\phi) R_{1}+\phi R_{3}-1}-\gamma_{1} R_{1} .
$$


The uniqueness of $R_{1}^{*}$ is due to the right hand side of (4.1) being a decreasing function of $R_{1}$ on this interval.

(3) If $1<R_{3}<1 / \phi$ and $R_{2} \geq R_{1}^{a}$ then there is only one supercritical endemic equilibrium for all $R_{1} \in\left(R_{1}^{a}, \infty\right)$.

(4) If $R_{3}<1$ then there is only one supercritical endemic equilibrium for all $R_{1} \in\left(R_{1}^{a}, \infty\right)$.

Proof See Appendix.

The above covers all possible regions of the parameter space $P$, with the exception of a finite number of special case equalities, such as $R_{3}=1$. If $R_{3}=1$, for example, then the preceding analysis is not sufficient to tell us whether or not there exist three supercritical endemic equilibria. We would need, in these special cases, to consider higher order terms in $b$. Again, we may extend the analysis of the proof to cover the case $R_{3}=1$ if required. Further details are given in Griffiths (2007).

Although the proofs of Theorems 4.1 and 4.2 assume that $\phi>0$, the results are also valid for $\phi=0$, with the obvious interpretations. Again this is shown in Griffiths (2007).

Our calculations have been based on the assumption that $b$ is small. It is interesting to see what happens therefore when we take this one stage further and set $b=0$. In this case we do not observe backward bifurcation. There is a unique endemic equilibrium given by

$$
\left(s_{1}^{*}, i_{1}^{*}, s_{2}^{*}, i_{2}^{*}, s_{3}^{*}, i_{3}^{*}\right)=\left(0,0,0,0, \frac{\beta_{3}}{\gamma_{2} \alpha_{3}}, 1-\frac{\beta_{3}}{\gamma_{2} \alpha_{3}}\right) .
$$

This endemic equilibrium is possible if and only if $R_{3}>1$.

\section{Limited consideration of the stability of the endemic equilibria}

We can eliminate one of the equations in (2.1) by setting $s_{3}=1-\left(s_{1}+s_{2}+i_{1}+i_{2}+i_{3}\right)$. However this leads to a characteristic equation which is a quintic and in general it is difficult to draw analytical conclusions, although it is possible to deduce that the unique endemic equilibrium is always LAS in the cases $\phi=1$ or $b=0$. As analytical results seem elusive instead we used numerical methods to analyse stability using both R (2004) and SOLVER (Gurney et al. (1996)). This involved both simulations and the application of the Routh-Hurwitz criteria. We have found some interesting stability patterns, which could form the basis of a future paper.

\section{A consideration of the possible bifurcation diagrams}

We consider values of $\phi$ with $0 \leq \phi<1$. We discuss the possible shapes of the curves that may arise on the bifurcation diagrams when using $\alpha_{1}$ as a bifurcation parameter. The endemic equilibrium solutions correspond to the positive roots of equation (3.2). 
If we keep all of the parameters fixed other than $\alpha_{1}$ then we may regard the solutions of this equation as functions of $\alpha_{1}$, and hence write them as $I\left(\alpha_{1}\right)$. Thus

$$
A I\left(\alpha_{1}\right)^{3}+B I\left(\alpha_{1}\right)^{2}+C I\left(\alpha_{1}\right)+D=0
$$

defines, implicitly, a curve of $I$ against $\alpha_{1}$. We shall refer to this as a bifurcation curve. We now differentiate both sides of the equation with respect to $\alpha_{1}$ to obtain

$$
\left(3 A I^{2}+2 B I+C\right) \frac{d I}{d \alpha_{1}}-b\left(b+\beta_{2}\right) 1-\phi I^{2}+\frac{b}{\gamma_{1}}+\frac{b+\beta_{3}}{\gamma_{2}} I+\frac{b\left(b+\beta_{3}\right)}{\gamma_{1} \gamma_{2}}=0,
$$

giving us

$$
\frac{d I}{d \alpha_{1}}=\frac{b\left(b+\beta_{2}\right) 1-\phi I^{2}+\frac{b}{\gamma_{1}}+\frac{b+\beta_{3}}{\gamma_{2}} I+\frac{b\left(b+\beta_{3}\right)}{\gamma_{1} \gamma_{2}}}{3 A I^{2}+2 B I+C},
$$

noting that this derivative is a function of both $I$ and $\alpha_{1}$, since $B$ and $C$ are both functions of $\alpha_{1}$. We know that there is a bifurcation point when $R_{0}=1$, i.e., when

$$
\alpha_{1}=\left(1-\frac{\gamma_{2} \alpha_{3} \phi}{b+\beta_{3}}\right) \frac{b+\beta_{1}}{1-\phi}
$$

and $I=0$. The sign of $d I / d \alpha_{1}$ at this point will indicate to us the possibility for backward bifurcation. We have

where

$$
\left.\frac{d I}{d \alpha_{1}}\right|_{R_{0}=1, I=0}=\frac{b^{2}\left(b+\beta_{2}\right)\left(b+\beta_{3}\right)(1-\phi)}{\gamma_{1} \gamma_{2} C\left(R_{0}=1\right)},
$$

$$
\begin{aligned}
C\left(R_{0}=1\right)=\frac{b\left(b+\beta_{1}\right)\left(b+\beta_{2}\right)\left(b+\beta_{3}\right)}{\gamma_{1} \gamma_{2}}-\frac{b \beta_{1}\left(b+\beta_{3}\right) \alpha_{2}(1-\phi)}{\gamma_{2}} \\
+\frac{\alpha_{3} \phi b\left(b+\beta_{1}\right)\left(b+\beta_{2}\right)}{\gamma_{1}}\left(\frac{b \gamma_{2}}{b+\beta_{3}}-1\right) .
\end{aligned}
$$

Backward bifurcation will occur when $C\left(R_{0}=1\right)<0$ (see the discussion following the proof of Theorem 3.2),

$$
\begin{aligned}
& \text { i.e., when } \frac{b \beta_{1}\left(b+\beta_{3}\right) \alpha_{2}(1-\phi)}{\gamma_{2}}+\frac{\alpha_{3} \phi b\left(b+\beta_{1}\right)\left(b+\beta_{2}\right)}{\gamma_{1}} \\
& \qquad>\frac{b\left(b+\beta_{1}\right)\left(b+\beta_{2}\right)\left(b+\beta_{3}\right)}{\gamma_{1} \gamma_{2}}+\frac{\alpha_{3} \phi b^{2}\left(b+\beta_{1}\right)\left(b+\beta_{2}\right)}{\gamma_{1}} \frac{\gamma_{2}}{b+\beta_{3}} .
\end{aligned}
$$

A typical bifurcation curve for this situation is given in Section 6.1 as Figure 6.1.1, although we note that it is also possible for the situation in Figure 6.1.2 to arise in this case. 
A simpler type of curve, as in Figure 6.1.3, could arise when $C\left(R_{0}=1\right)>0$. Here there is forward bifurcation. For many relatively simple epidemic models this is the only type of bifurcation curve that can result, for which there is always a unique supercritical endemic equilibrium but where there is no possibility for subcritical endemic equilibria to exist. We note, however, that in our case this does not preclude the possibility that two subcritical endemic equilibria exist when $C\left(R_{0}=1\right)>0$. Indeed, the preceding analysis has already shown that this is possible. In this case the bifurcation curve could be of the type shown in Figure 6.1.4 or Figure 6.1.5 (noting that, by continuity, there will necessarily then be three endemic equilibria for some region of the parameter space for which $R_{0}>1$ ). The bifurcation curve in Figure 6.1.6 may also be possible, in which there exist three endemic equilibria for some region of the parameter space such that $R_{0}>1$, but for which no backward bifurcation is exhibited.

All of the bifurcation diagrams shown in Figures 6.1.1 to 6.1.6 pos sess either zero, one or two vertical turning points. Where present, these turning points are denoted on the diagrams by $R_{1}^{\min }$ and/or $R_{1}^{\max }$, alternatively $\alpha_{1}^{\min }$ and/or $\alpha_{1}^{\max }$ when using $\alpha_{1}$ as the bifurcation parameter. We show below that it is in fact possible to calculate the exact values of $\alpha_{1}^{\min }$ and/or $\alpha_{1}^{\max }$. We now consider whether there is the potential for more than two vertical turning points, which would give rise to more complicated bifurcation diagrams.

Firstly, let us rearrange the equation (6.1) in the form

$$
\alpha_{1}=\frac{A I^{3}+\widetilde{B} I^{2}+\widetilde{C} I+\widetilde{D}}{b\left(b+\beta_{2}\right)(1-\phi) I^{2}+\frac{b}{\gamma_{1}}+\frac{b+\beta_{3}}{\gamma_{2}} I+\frac{b\left(b+\beta_{3}\right)}{\gamma_{1} \gamma_{2}}},
$$

where

$$
\begin{aligned}
& \widetilde{B}=\left(b+\beta_{1}\right)\left(b+\beta_{2}\right) b+\frac{b}{\gamma_{1}}+\frac{b+\beta_{3}}{\gamma_{2}}-\alpha_{2} \beta_{1} b(1-\phi)-\alpha_{3} \beta_{1} \beta_{2}(1-\phi)-\alpha_{3} \phi\left(b+\beta_{1}\right)\left(b+\beta_{2}\right), \\
& \widetilde{C}=b\left(b+\beta_{1}\right)\left(b+\beta_{2}\right) \frac{b}{\gamma_{1}}+\frac{b+\beta_{3}}{\gamma_{2}}+\frac{b+\beta_{3}}{\gamma_{1} \gamma_{2}}-\alpha_{2} \beta_{1} \frac{b+\beta_{3}}{\gamma_{2}}(1-\phi)-\alpha_{3} \phi\left(b+\beta_{1}\right)\left(b+\beta_{2}\right) 1+\frac{1}{\gamma_{1}}
\end{aligned}
$$

and

$$
\widetilde{D}=\frac{b^{2}}{\gamma_{1} \gamma_{2}}\left(b+\beta_{1}\right)\left(b+\beta_{2}\right)\left(b+\beta_{3}\right)\left(1-\frac{\alpha_{3} \phi \gamma_{2}}{b+\beta_{3}}\right) \text {. }
$$

We then have

$$
\frac{d \alpha_{1}}{d I}=\frac{F(I) 3 A I^{2}+2 \widetilde{B} I+\widetilde{C}-b\left(b+\beta_{2}\right) 2 I+\frac{b}{\gamma_{1}}+\frac{b+\beta_{3}}{\gamma_{2}}(1-\phi)\left(A I^{3}+\widetilde{B} I^{2}+\widetilde{C} I+\widetilde{D}\right)}{F(I)^{2}},
$$

where $F(I)=b\left(b+\beta_{2}\right)(1-\phi) I^{2}+\frac{b}{\gamma_{1}}+\frac{b+\beta_{3}}{\gamma_{2}} I+\frac{b\left(b+\beta_{3}\right)}{\gamma_{1} \gamma_{2}}$, noting that $F(I)^{2}>0$ when $I>0$ and $\phi<1$. The values of $I$ corresponding to the vertical turning points on the bifurcation diagram are the real positive solutions of the quartic equation

$$
F(I) 3 A I^{2}+2 \widetilde{B} I+\widetilde{C}-b\left(b+\beta_{2}\right) 2 I+\frac{b}{\gamma_{1}}+\frac{b+\beta_{3}}{\gamma_{2}}(1-\phi)\left(A I^{3}+\widetilde{B} I^{2}+\widetilde{C} I+\widetilde{D}\right)=0 .
$$

In order to obtain the values of $\alpha_{1}$ at these turning points we substitute the real positive solutions of the above quartic back into 


$$
\alpha_{1}=\frac{A I^{3}+\widetilde{B} I^{2}+\widetilde{C} I+\widetilde{D}}{b\left(b+\beta_{2}\right)(1-\phi) I^{2}+\frac{b}{\gamma_{1}}+\frac{b+\beta_{3}}{\gamma_{2}} I+\frac{b\left(b+\beta_{3}\right)}{\gamma_{1} \gamma_{2}}},
$$

although see the caveat in (2) below in this regard.

Before proceeding further, there are several things that are worth noting at this stage, with regard to the above equation:

(1) On restricting the domain to non-negative values of $I$, it defines the bifurcation curve. In actual fact bifurcation diagrams tend to be drawn with the bifurcation parameter on the horizontal axis, so the usual convention of drawing the graph of $y=f(x)$ so that $x$ is on the horizontal axis would give us the bifurcation curve 'on its side'. In other words, the bifurcation curve is given by the graph, restricted to the positive quadrant, of the above equation.

(2) We see that $\alpha_{1}$ is a rational function of $I$. In fact $\alpha_{1}=s(I) / t(I)$ where $s$ is a cubic and $t$ is a quadratic. This function is defined for all non-negative values of $I$ since $t$ is non-zero (more specifically, positive) for all $I \geq 0$. This means that the curve defined by $\alpha_{1}=s(I) / t(I)$ will not possess any vertical asymptotes when $I \geq 0$, and will thus be continuous for these values of $I$, although there may exist positive values of $I$ for which $s(I) / t(I)$ is negative (see Figures 6.1.2 and 6.1.4). So, in calculating $\alpha_{1}^{\min }$ via the method given above, it is possible that a negative value would result.

(3) The bifurcation curve has an oblique asymptote with equation

$$
I=\frac{b(1-\phi)}{b+\beta_{1}} \alpha_{1}
$$

From the above we see that, in Figures 6.1.1, 6.1.4, 6.1.5 and 6.1.6, we would be able to calculate the exact values of $R_{1}^{\min }$ and/or $R_{1}^{\max }$ by performing a similar procedure with $R_{1}$ instead of $\alpha_{1}$, noting that it is possible to obtain (via radicals) the exact solutions to any quartic equation.

The fact that the number of vertical turning points on our bifurcation diagram is determined by the number of real positive solutions to a quartic may lead us to believe that it might be possible to obtain bifurcation curves that possess three or even four turning points. However, we now show that neither of these situations is possible.

Theorem 6.1 The bifurcation diagrams for the epidemic model we are currently considering cannot have more than two vertical turning points.

Proof We may write (6.2) in the form $\alpha_{1}=\frac{a\left(I^{3}+b I^{2}+c I+d\right)}{I^{2}+e I+f}$ where, because of the parameter expressions they represent, $a, e$ and $f$ must be positive. We then have

$$
\frac{d \alpha_{1}}{d I}=\frac{a I^{4}+2 e I^{3}+(3 f+b e-c) I^{2}+2(b f-d) I+(c f-d e)}{I^{2}+e I+f^{2}},
$$


and we are thus looking for the real positive solutions of

$$
I^{4}+2 e I^{3}+(3 f+b e-c) I^{2}+2(b f-d) I+(c f-d e)=0 .
$$

A quartic equation with real coefficients and at least three positive real roots must actually possess four real roots, $s, t, u$ and $v$ say. Now

$$
\begin{array}{r}
(I-s)(I-t)(I-u)(I-v)=I^{4}-(s+t+u+v) I^{3}+(s t+s u+s v+t u+t v+u v) I^{2} \\
-(s t u+s t v+s u v+t u v) I+s t u v .
\end{array}
$$

Let us compare the coefficients of this quartic expression with (6.4).

We see immediately that four turning points are not possible, since in that case we would have $2 e=-(s+t+u+v)<0$.

Now say that there are three turning points. Then three of the roots $s, t, u$ and $v$ are positive, while the remaining one is non-positive. Say that $s$ is the non-positive root. Since we require $2 e=-(s+t+u+v)>0$ we see that $-s>t+u+v$. Then $s t u v<0, s t+s u+s v+t u+t v+u v<0$ and $s t u+s t v+s u v+t u v<0$.

The above tells us that

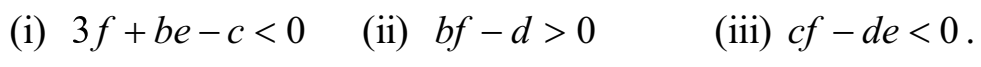

Thus from (ii) $b>d / f$. Moreover $c>b e+3 f>d e / f+3 f>c+3 f$. This is a contradiction, thereby completing the proof of the Theorem.

\subsection{Possible shapes of bifurcation diagrams}

We consider here the possible shapes of the bifurcation curves discussed in the previous section. These will depend on the existence of $R_{1}^{\min }$ and/or $R_{1}^{\max }$ (alternatively $\alpha_{1}^{\min }$ and/or $\alpha_{1}^{\max }$ ) and, should either of them exist, their values. However, although we have demonstrated how to calculate these numbers analytically, we would also like to gain an idea of the regions of the parameter space $P$ that lead to particular diagrams. We use the inequalities obtained in Section 4 with $0<\phi<1$ to classify the bifurcation diagrams, bearing in mind that the various shapes result under the given conditions on the parameters provided that $b$ is sufficiently small.

FIGURE 6.1.1

$$
R_{3}<1 \text { and } \frac{1-\phi R_{3}}{1-\phi}<R_{2}<\frac{1}{1-\phi}\left(1+\gamma_{1}\right)\left(1-\phi R_{3}\right)+2 \sqrt{\gamma_{1}\left(1-R_{3}\right) 1-\phi R_{3}} .
$$

\section{FIGURE 6.1.2}

$$
\begin{array}{ll} 
& 1<R_{3}<\frac{1}{\phi} \text { and } R_{2} \geq \frac{1-\phi R_{3}}{1-\phi} \\
\text { or } \quad R_{3}<1 \text { and } R_{2} \geq \frac{1}{1-\phi}\left(1+\gamma_{1}\right)\left(1-\phi R_{3}\right)+2 \sqrt{\gamma_{1}\left(1-R_{3}\right) 1-\phi R_{3}} .
\end{array}
$$


FIGURE 6.1.3

$$
\begin{aligned}
& R_{3}>\frac{1}{\phi} \\
& \text { or } \quad R_{3}<1 \text { and } R_{2} \leq \frac{1-\phi R_{3}}{1-\phi} .
\end{aligned}
$$

FIGURE 6.1.4

$$
1<R_{3}<\frac{1}{\phi} \text { and } R_{2}<\frac{1-\phi R_{3}}{1-\phi} .
$$

The bifurcation diagrams in Figures 6.1.1 to 6.1.3 are similar to the ones obtained for the two-stage model studied by Greenhalgh et al. (2000), in which backward bifurcation is and is not present respectively. In our case Figure 6.1.1 corresponds to the situation in which there exist two subcritical endemic equilibria but only one supercritical endemic equilibrium. Figure 6.1.3 demonstrates the situation in which there are no multiple equilibria at all. Figures 6.1.4, 6.1.5 and 6.1.6 display more complicated bifurcation curves that cannot arise in the two-stage model.

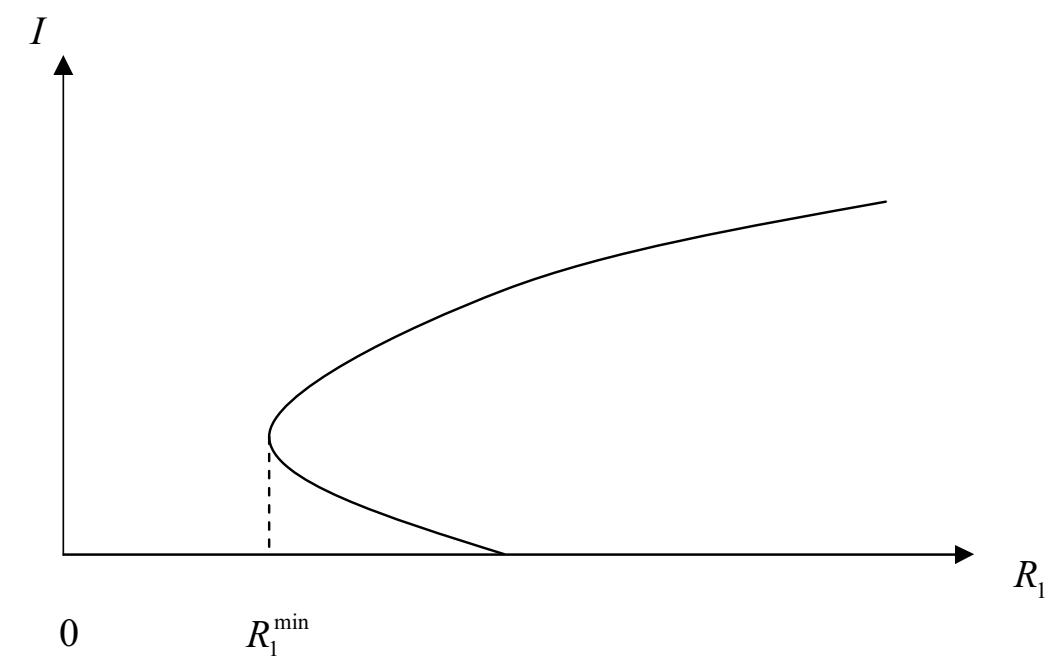

FIGURE 6.1.1: A bifurcation diagram exhibiting backward bifurcation.

Note that in Figure 6.1.2, once the disease is established it cannot be eradicated by simply decreasing the value of the bifurcation parameter. On the other hand, the bifurcation curve in Figure 6.1.3 is the type observed in most simple models, for which there are no subcritical endemic equilibria and exactly one supercritical endemic equilibrium. 


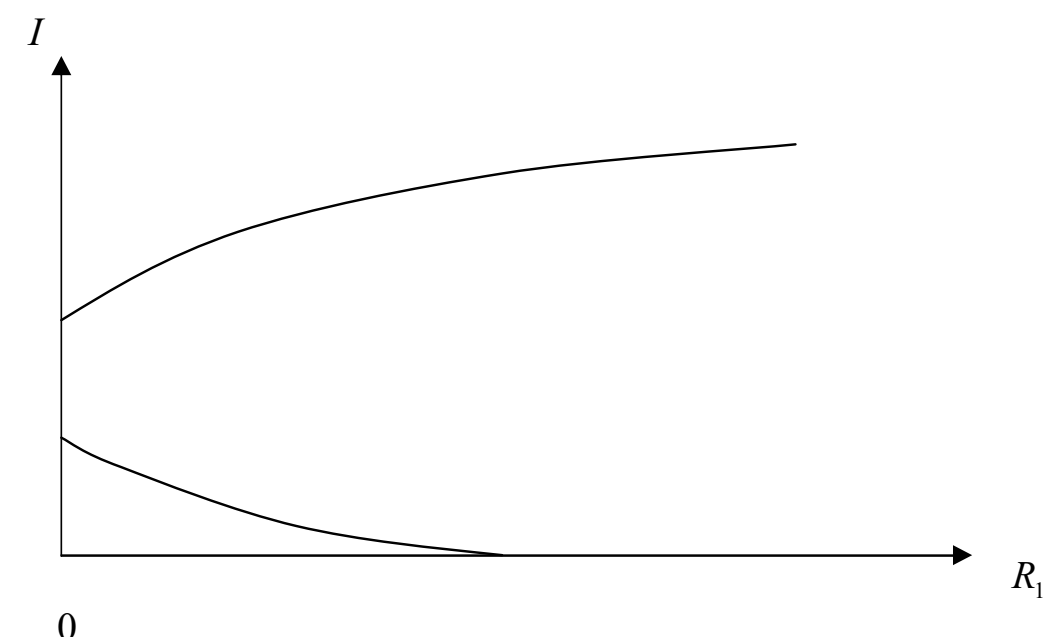

FIGURE 6.1.2: A bifurcation diagram exhibiting backward bifurcation. There are endemic equilibria present for all positive values of $R_{1}$ (and hence also for all positive values of $R_{0}$ ).

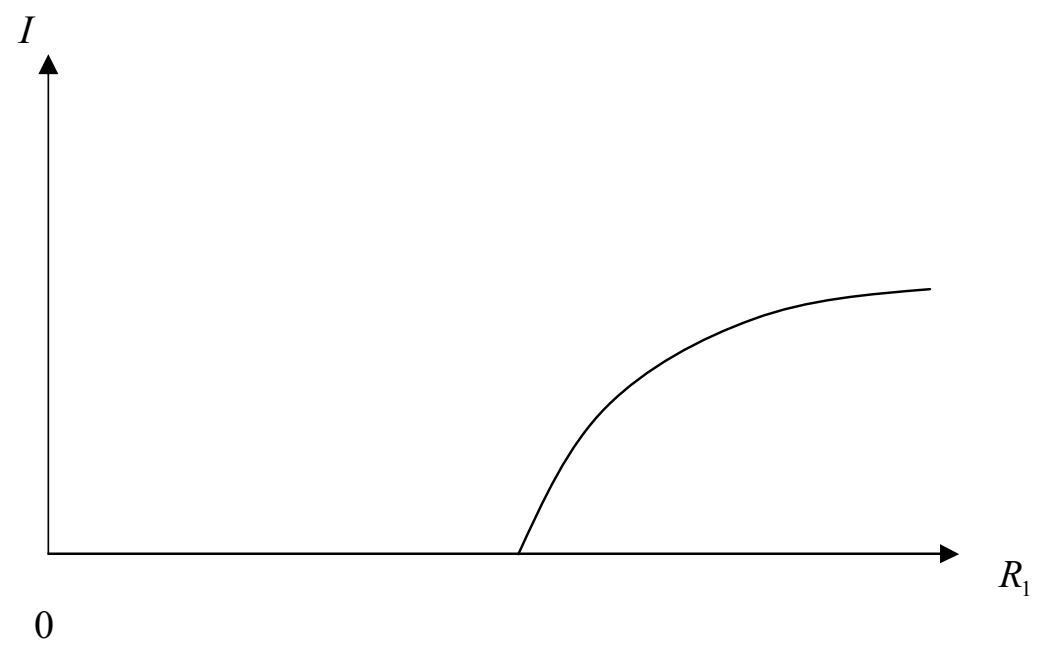

FIGURE 6.1.3: A bifurcation diagram exhibiting forward bifurcation. There is a unique supercritical endemic equilibrium. 


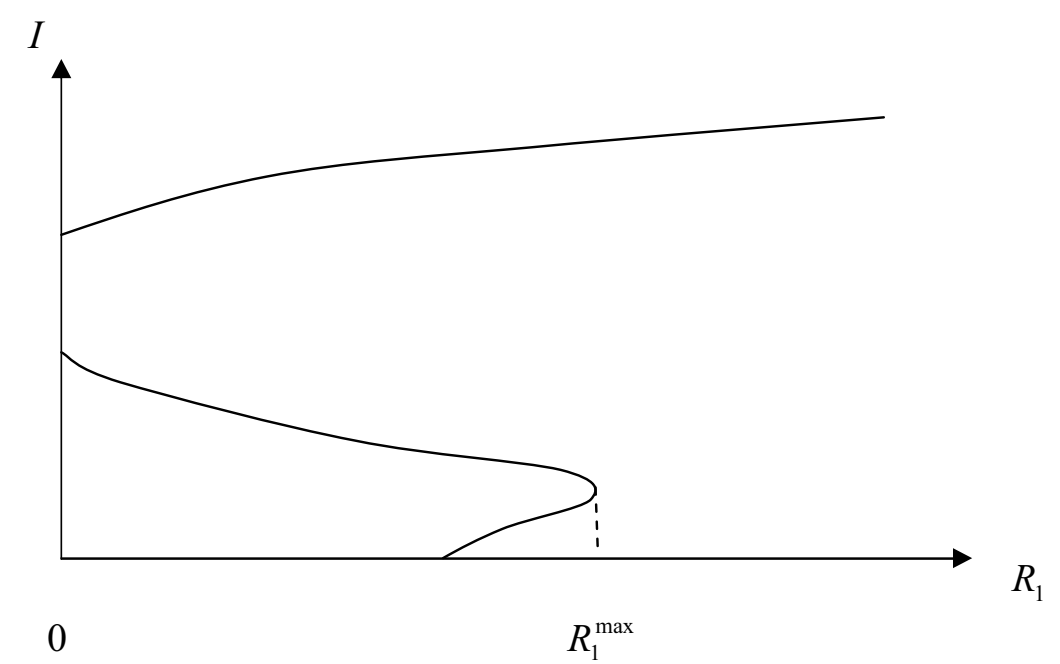

FIGURE 6.1.4: A bifurcation diagram exhibiting forward bifurcation as well as multiple subcritical and supercritical endemic equilibria. There are endemic equilibria for all positive values of $R_{1}$ (and hence for all positive values of $R_{0}$ ).

There are two more obvious candidates for the possible shapes of our bifurcation curves. These are given in Figures 6.1.5 and 6.1.6 below. All that we can say here is that the bifurcation curves given in Figures 6.1.5 and 6.1.6 cannot occur if $b$ is sufficiently small, although we note here that there are degenerate points where, as has been previously pointed out, we cannot be certain what happens, so we cannot strictly rule out Figures 6.1.5 and 6.1.6 for these degenerate parameter values.

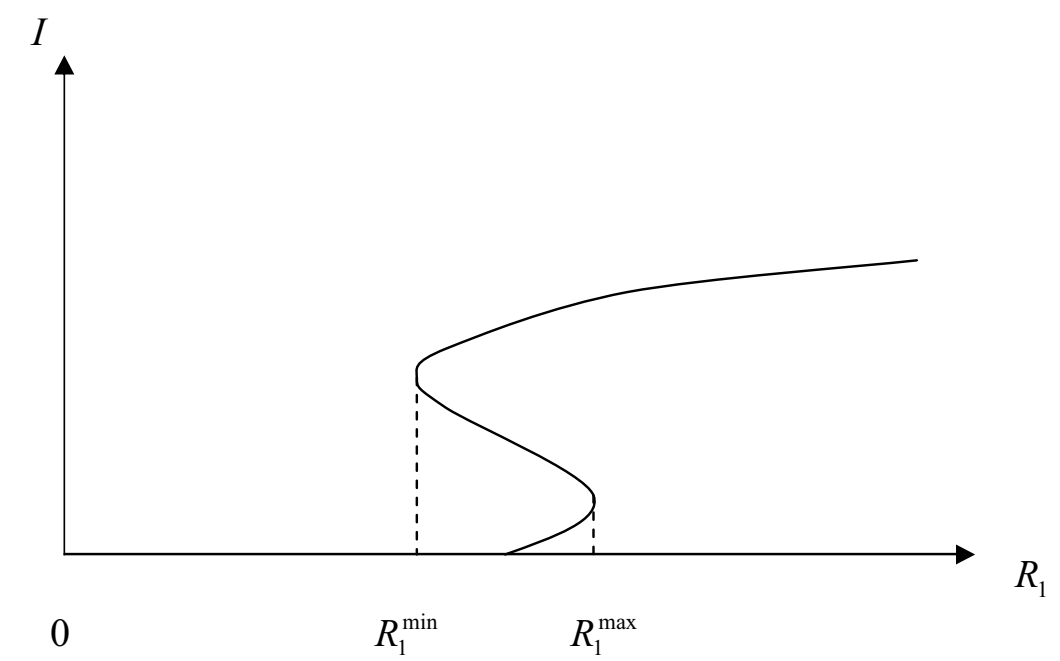

FIGURE 6.1.5: A bifurcation diagram exhibiting forward bifurcation as well as multiple subcritical and supercritical endemic equilibria. There exists a positive value of $R_{1}, R_{1}^{\mathrm{min}}$, below which no endemic equilibria are possible. 


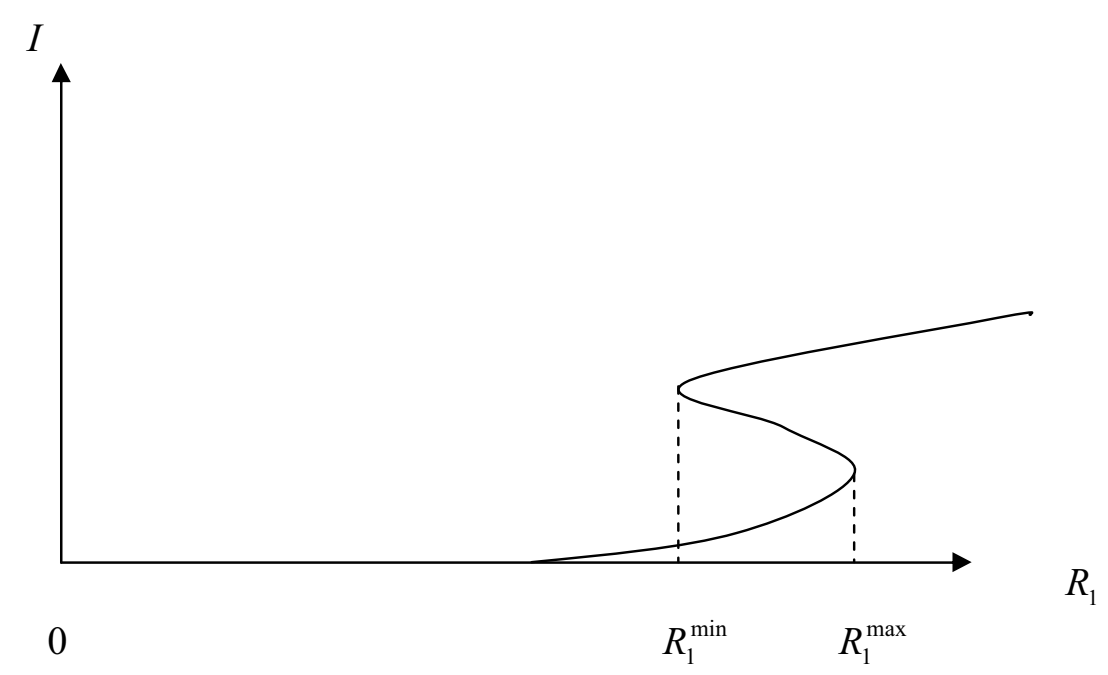

FIGURE 6.1.6: A bifurcation diagram exhibiting forward bifurcation as well as multiple supercritical endemic equilibria, but no subcritical endemic equilibria.

Despite this limitation, the analysis we have carried out with $b$ small does demonstrate the existence of at least four differently-shaped bifurcation curves, and does not preclude the existence of a further two. We do, however, plot some bifurcation curves in the next section, and, guided by the conditions that we have obtained for two or three endemic equilibria to exist for sufficiently small $b$, it turns out to be very easy to find regions in the parameter space that give bifurcation curves as in Figures 6.1.5 and 6.1.6.

\subsection{Plotted bifurcation diagrams}

It turns out that the sets of conditions obtained in Section 4 in order to classify the shapes of the bifurcation diagrams do not generally require $b$ to be unrealistically small. This may be noted by considering the various bifurcation diagrams plotted in the remainder of this section, along with their corresponding sets of parameter values. In fact, prior knowledge of the shapes of these diagrams was, in each case, acquired by using the sets of inequalities given in Section 4.

We now show that it is possible to find sets of parameter values that give bifurcation diagrams of the types shown in Figures 6.1.5 and 6.1.6. The bifurcation diagram in Figure 6.2.1(a) is similar to the one shown in Figure 6.1.5. It was obtained for the parameter values given below:

$$
b=0.001, \alpha_{2}=0.03, \alpha_{3}=0.255, \beta_{1}=\beta_{2}=\beta_{3}=0.1, \gamma_{1}=\gamma_{2}=0.5 \text { and } \phi=0.001 \text {, }
$$

where the parameters $\gamma_{1}, \gamma_{2}$ and $\phi$ are dimensionless, whilst the remainder all possess the units days ${ }^{-1}$. These parameters were motivated by realistic parameter values used for BRSV. We kept our parameter values similar to those used by Greenhalgh et al. (2000) in their two-stage BRSV model, who had themselves used values based on the estimates given by de Jong et al. (1996). 


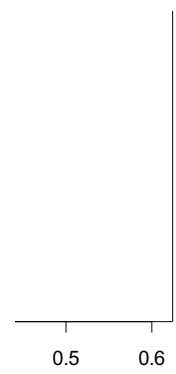

(a)

(b)

FIGURE 6.2.1: (a) A bifurcation diagram exhibiting both multiple subcritical and supercritical endemic equilibria. (b) A bifurcation diagram exhibiting multiple supercritical endemic equilibria but no subcritical endemic equilibria.

We can see that both multiple subcritical and supercritical endemic equilibria are present. All bifurcation diagrams were plotted by writing programmes in R. In all bifurcation diagrams a solid line represents a locally stable endemic equilibrium and a dotted line an unstable equilibrium.

On the other hand, the bifurcation diagram in Figure 6.2.1(b) arose from the following parameter values, where the units are the same as those above:

$$
b=0.001, \alpha_{2}=0.03, \alpha_{3}=0.238, \beta_{1}=\beta_{2}=\beta_{3}=0.1, \gamma_{1}=\gamma_{2}=0.5 \text { and } \phi=0.001 \text {. }
$$

It is similar in shape to the bifurcation diagram shown in Figure 6.1.6. There are multiple supercritical endemic equilibria but no subcritical endemic equilibria present here.

It should be noted that our bifurcation diagrams have the variable $I$ along the vertical axis, rather than any of the variables $i_{1}^{*}, i_{2}^{*}$ or $i_{3}^{*}$. Also, the curves on bifurcation diagrams utilising $i_{1}, i_{2}$ or $i_{3}$ have a horizontal asymptote by virtue of the fact that each of these variables are bounded above by 1 . However, since $I=\alpha_{1} i_{1}^{*}+\alpha_{2} i_{2}^{*}+\alpha_{3} i_{3}^{*}$, the bifurcation diagrams for $I$ have oblique asymptotes instead. We gave this oblique asymptote in equation (6.3).

It is interesting to see an example of the way that a bifurcation diagram changes if, starting from the type as in Figure 6.1.4, we gradually increase $b$. A program was written in $\mathrm{R}$ to produce dynamic bifurcation diagrams. These are animated diagrams demonstrating how the bifurcation curve changes as one of the parameters, other than the bifurcation parameter, is varied. The diagrams in Figures 6.2.2(a), (b), (c) and (d) show snapshots of such a dynamic bifurcation diagram with $b$ as the dynamic parameter. The remaining parameter values were

$$
\alpha_{2}=0.03, \alpha_{3}=0.24, \beta_{1}=\beta_{2}=\beta_{3}=0.1, \gamma_{1}=\gamma_{2}=0.5 \text { and } \phi=0.3 \text {. }
$$


It can be seen that as $b$ increases we go from the type of bifurcation diagram in Figure 6.1.4 to those in Figures 6.1.5 and 6.1.6.

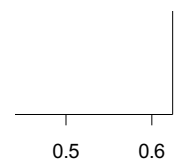

(a)

(b)

(c)

(d)

FIGURE 6.2.2: A series of bifurcation diagrams showing how the shape can change as $b$ is increased.

6.3 A bifurcation diagram demonstrating multiple endemic equilibria for realistic parameter values

It could be argued that, in order for our model to be biologically meaningful, the infectivity levels should be such that $\alpha_{1}>\alpha_{2}>\alpha_{3}$. In other words, first-time infected individuals are more effective at spreading the infection than are second-time infected individuals who, in turn, are more effective at spreading the infection than are third-time infected individuals. 
By using our conditions for the existence of two endemic equilibria, we obtained the bifurcation diagram below with the following set of parameter values

$$
\begin{gathered}
b=0.001, \alpha_{2}=0.05, \alpha_{3}=0.04, \beta_{1}=0.07, \beta_{2}=0.08, \beta_{3}=0.025, \gamma_{1}=1.0, \\
\gamma_{2}=0.9 \text { and } \phi=0.0,
\end{gathered}
$$

where $\gamma_{1}, \gamma_{2}$ and $\phi$ are dimensionless, while the rest of the parameters possess the units days $^{-1}$. It can be seen, from Figure 6.3.1, that two subcritical endemic equilibria do exist for some values of $\alpha_{1}$ greater than 0.05 . Three supercritical endemic equilibria are also possible.

FIGURE 6.3.1: A bifurcation diagram to illustrate the fact that multiple subcritical and supercritical endemic equilibria are possible for what are arguably a realistic set of parameter values.

Greenhalgh et al. (2000) give a stability result for the two-stage BRSV model that is equivalent to stating that a particular endemic equilibrium is LAS if, and only if, the gradient of the bifurcation curve at that point is positive. On considering the bifurcation diagrams in this section it might appear that the same could be said for the three-stage model, but this turns out not to be the case. We have obtained bifurcation diagrams for which sections of the curve possess a positive gradient yet display instability there. Stability is thus clearly a far more complex issue in the three-stage model. It is interesting to note, on considering Figure 6.2.2(b) for example, that multiple LAS endemic equilibria are possible.

\section{Conclusions}

We have extended, in a natural way, a deterministic epidemic model for which backward bifurcation was already known to be present. The original two-stage model allows for the possibility that individuals who recover from the disease but 
subsequently become re-infected may, as a result of partial immunity, possess different characteristics from first-time infected individuals in terms of their recovery and infectivity. We argued that the inclusion of a third stage might further enhance its realism. Our main purpose has been to investigate the potential for more complicated bifurcation diagrams to exist. Such diagrams might allow us to foresee, for certain regions of the parameter space, some particularly interesting dynamic behaviour in epidemic models such as subcritical invasions, and could also have repercussions with regard to disease control.

By performing a detailed equilibrium analysis of the extended model, we have found that there is indeed the possibility for the appearance of some unusual bifurcation curves. We have been able to classify these bifurcation diagrams geometrically, in terms of the regions of the parameter space in which they occur. In particular, we have found bifurcation diagrams for which subcritical endemic equilibria occur, even in the presence of forward bifurcation. We have also demonstrated the possibility for multiple supercritical endemic equilibria to exist. Furthermore, there is the potential for such diagrams to exist with realistic parameter values. We have also been able to say something about the types of bifurcation curve that definitely may not occur for our model.

As a consequence of the above, our investigations would appear to shed light on the potential dynamics of diseases such as BRSV and Aujesky's disease in which resistance to infection is gained in several stages. Indeed we have shown that complex bifurcation diagrams are possible with parameter values based on BRSV. There are also potential implications for control of these diseases, as the proportion $\phi$ vaccinated at birth is a key parameter in determining the shape of the bifurcation diagrams. As discussed by Greenhalgh et al. (2000), de Jong et al. (1996) build a stochastic model for BRSV and estimate parameters from serological data on antibodies against BRSV in sera from cattle in six dairy herds. They conclude that persistence of the infection amongst seropositive cattle is still plausible although noone has yet succeeded in isolating the virus in re-infected cattle. The model is equally applicable to Aujesky's disease in pigs. De Jong and Kimman (1994) and Sabó and Blaškovič (1970) obtained experimental evidence that the two-stage model is more suitable for this disease than a single stage model.

Bearing in mind the comments made towards the beginning of Section 4, the building blocks of our three-stage model are essentially three modified SIS submodels. From this it becomes clear that our model may be regarded as a series of three interconnected one-stage submodels, two of which are similar to SIR and the other is SIS. The presence, or otherwise, of backward bifurcation will depend in a very sensitive way on the interaction between them. Furthermore, whether or not a subcritical invasion of the disease actually takes place is sensitive both to the number of infected individuals introduced into the disease free population and to the initial ratio of the types of these individuals.

The conditions for the different shapes of bifurcation diagram which can occur are parsimoniously cast in terms of the easily biologically identifiable parameters $R_{1}$, $R_{2}$ and $R_{3}$, the limiting basic reproduction numbers for the three submodels, $\phi$, the vaccination proportion at birth and the infectivity reduction factor $\gamma_{1}$. It does not seem easy to give a more concise biological explanation of precisely when different types of bifurcation diagram are possible. However, we might consider the situation whereby all of the parameters are kept fixed except for $\alpha_{1}, \alpha_{2}$ and $\alpha_{3}$, for example. 
In a classical epidemic model reducing $R_{0}$ to below one is sufficient to eliminate the disease. For our model this tendency can be counteracted if $R_{2}$ or $R_{3}$ are sufficiently large. In these cases disease can persist in the population when $R_{0}=1$ due to backward bifurcation. When this happens we may regard one of the submodel infection processes as in some sense playing a dominant role with regard to the overall dynamics of the system. Of course, for a realistic model we might expect $\alpha_{1}>\alpha_{2}>\alpha_{3}$. Note, however, that such a condition does not preclude the possibility for backward bifurcation. For example, since $R_{3}=\alpha_{3} / \beta_{3}$ then even if $\alpha_{3}$ is not particularly large it is still possible that $R_{3}$ is large enough for backward bifurcation provided that $\beta_{3}$ is sufficiently small. Indeed, Greenhalgh et al. (2000) note that the average length of subsequent infectious periods in BRSV appear to exceed that of the first.

There is scope for plenty of further work in this area. Firstly, a more detailed stability analysis could be carried out, both analytically and numerically. From this we would be able to consider in more depth the possibilities for eradicating the disease from the population. Our initial numerical investigations have revealed that the situation is considerably more complex than was the case for the two-stage BRSV model in that the upper branch of the bifurcation curve is not now necessarily LAS along its entire length.

Secondly, we can investigate further epidemic models to see whether refining or extending them leads to the similar sort of phenomena that we have demonstrated in this paper. Again here, our preliminary studies in this direction corroborate many of the findings presented here, and have also unearthed some apparently new stability phenomena.

Finally, we have already started looking at stochastic versions of these models in order to consider possible connections between the presence of the more unusual bifurcation curves and any unexpected stochastic phenomena that we observe. In particular, we have found that a very interesting avenue of enquiry has been to consider whether we can relate in any way the deterministic concept of a bifurcation curve to the stochastic concept of the probability of extinction of the disease, given that one infected person is introduced into the population at the DFE.

\section{References}

C. Castillo-Chavez, K. Cooke, W. Huang and S. A. Levin. Results on the dynamics for models for the sexual transmission of the human immunodeficiency virus. Applied Mathematics Letters, 2:327-331, $1989 a$.

C. Castillo-Chavez, K. Cooke, W. Huang and S. A. Levin. The role of long incubation periods in the dynamics of HIV/AIDS. Part 2: Multiple group models. Mathematical and Statistical Approaches to AIDS E pidemiology, Volume 83, Lecture Notes in Biomathematics, 200-217. Springer-Verlag, $1989 b$.

M. C. M. de Jong, T. G. Kimman. Experimental quantification of vaccine induced reduction in virus transmission. Vaccine, 8:761-766, 1994. 
M. C. M. de Jong, W. H. M. Van der Poel, J. A. Kramps, A. Brand and J. T. Van Oirschot. Persistence and recurrent outbreaks of bovine respiratory syncytial virus on dairy farms. American Journal of Veterinary Research, 57:628-633, 1996.

O. Diekmann, J. A. P. Heesterbeek and J. A. J. Metz. On the definition and computation of the basic reproduction ratio $R_{0}$ in models for infectious diseases in heterogeneous populations. Journal of Mathematical Biology, 28:365-382, 1991.

J. Dushoff. Incorporating immunological ideas in epidemiological models. Journal of Theoretical Biology, 180:181-187, 1996.

D. Greenhalgh, O. Diekmann and M. C. M. de Jong. Subcritical endemic steady states in mathematical models for animal infections with incomplete immunity. Mathematical Biosciences, 165:1-25, 2000.

M. Griffiths. Backward Bifurcation and Associated Phenomena in Epidemic Models. Ph.D. Thesis. University of Strathclyde, 2007.

W. S. C. Gurney, S. Tobia and G. Watt. SOLVER: A programme template for initial value problems expressable as sets of coupled ordinary or delay differential equations. STAMS, University of Strathclyde, 1996.

K. P. Hadeler and C. Castillo-Chavez. A core group model for disease transmission. Mathematical Biosciences, 128:41-55, 1995.

K. P. Hadeler and P. van den Driessche. Backward bifurcation in epidemic control. Mathematical Biosciences, 146:15-35, 1997.

W. Huang, K. L. Cooke and C. Castillo-Chavez. Stability and bifurcation for a multiple-group model for the dynamics of HIV/AIDS transmission. SIAM Journal of Applied Mathematics, 52:835-854, 1992.

Q. J. A. Khan and D. Greenhalgh. Hopf bifurcation in epidemic models with a time delay in vaccination. IMA Journal of Mathematics Applied in Medicine and Biology, 16:113-142, 1999.

R Development Core Team. R: A language and environment for statistical computing. R Foundation for Statistical Computing, Vienna, Austria. ISBN 3 900051-00-3, URL http://www.R-project.org, 2004.

A. Sabó and D. Blaškovič. Resistance of tonsillary and throat mucosa to re-infection with a homologous pseudorabies virus strain. Acta Virologica, 14:17-24, 1970.

A. N. Schweitzer and R. M. Anderson. Dynamic interaction between CD4 ${ }^{+}$T-cells and parasitic helminths: Mathematical models of heterogeneity in outcome. Parasitology, 105:513-522, 1992.

P. van den Driessche and J. Watmough. A simple SIS epidemic model with a backward bifurcation. Journal of Mathematical Biology, 40:525-540, 2000. 
R. S. Varga. Matrix Iterative Analysis, Prentice-Hall, Englewood Cliffs, New Jersey, 1962.

\section{APPENDIX}

\section{Proof of Lemma 2.1.2}

$$
\begin{aligned}
& \begin{aligned}
\left(\frac{1}{a}+\frac{1}{b}+\frac{1}{c}\right) a & +b+c-d-e-f \\
& =3-\left(\frac{d}{a}+\frac{e}{b}+\frac{f}{c}\right)+a-d\left(\frac{1}{b}+\frac{1}{c}\right)+b-e\left(\frac{1}{a}+\frac{1}{c}\right)+c-f\left(\frac{1}{a}+\frac{1}{b}\right), \\
> & 2,
\end{aligned} \\
& \text { since }\left(\frac{d}{a}+\frac{e}{b}+\frac{f}{c}\right)<1 . \text { Hence }(a+b+c-d-e-f) \geq \frac{a b c}{a b+b c+c a} . \text { But } \\
& \frac{a b+b c+c a}{a b c} a b c-d b c-e a c-f a b<a b+b c+c a-d b+c-e a+c-f a+b .
\end{aligned}
$$

The result follows.

Proof of Lemma 3.1 Let us consider the graph of $y=f\left(x, \lambda_{d}\right)$. This passes through the origin, and, since $d y / d x=3 a x^{2}+2 b\left(\lambda_{d}\right) x+c\left(\lambda_{d}\right)$, we see that the gradient at the origin, $c\left(\lambda_{d}\right)$, is negative (by assumption). Then, since $a>0$, we have that $f\left(x, \lambda_{d}\right)=0$ has a positive root. Now, as we decrease $\lambda$ (and thus increase $d$ from $0)$, the fact the coefficients are continuous functions of $\lambda$ guarantees that there will be some open interval, $\left(\lambda_{1}, \lambda_{d}\right)$ say, for which $f(x, \lambda)=0$ has two positive real roots for all $d$ in $0, d\left(\lambda_{1}\right)$, thereby completing the proof.

Proof of Theorem 4.1 $R_{0}<1$ implies that $\hat{D}>0$ so that $F(I)=0$ possesses at least one real negative root and there will either be zero or two positive roots. In other words, there will either be zero or two subcritical endemic equilibria. For there to be any possibility at all for two endemic equilibria to exist, the graph of $y=F(I)$ must possess two distinct real turning points so we require that $\hat{B}^{2}>3 \hat{C}$.

Suppose that $\Delta=4 \hat{B}^{2}-3 \hat{C}>0$, and let $\lambda$ and $\mu$ be the distinct roots of $F^{\prime}(I)=0$. We have that $\lambda+\mu=-2 \hat{B} / 3$. Then, as $F^{\prime}(0)=\hat{C}$, we have, for example, that $\lambda$ and $\mu$ are both non-negative if, and only if, $\hat{B}<0$ and $\hat{C} \geq 0$. Now, by writing $\bar{\Delta}=F(\lambda) F(\mu)$, putting all of the above together and considering the possible shapes of the graph $y=F(I)$ we arrive at the following:

If $R_{0}<1$ there are two endemic equilibria if, and only if, either 


$$
\begin{array}{ll} 
& \Delta>0, \hat{B}<0, \hat{C} \geq 0 \text { and } \bar{\Delta}<0 \\
\text { or } & \Delta>0, \hat{C}<0 \text { and } \bar{\Delta}<0 .
\end{array}
$$

We have, since $\hat{B}=\beta_{3}\left(1-R_{3}\right) / \gamma_{2}+O(b)$ and $\hat{C}=O(b)$, that

$$
\frac{\Delta}{4}=\hat{B}^{2}-3 \hat{C}=\left(\frac{\beta_{3}}{\gamma_{2}}\left(1-R_{3}\right)\right)^{2}+O(b) .
$$

Also, as is shown by Khan and Greenhalgh (1999), we have

$$
\bar{\Delta}=\frac{4}{27} \hat{C}^{3}-\frac{1}{27} \hat{B}^{2} \hat{C}^{2}+\frac{4}{27} \hat{B}^{3} \hat{D}-\frac{2}{3} \hat{B} \hat{C} \hat{D}+\hat{D}^{2}
$$

from which, since $\hat{C}^{3}=O\left(b^{3}\right), \hat{B}^{2} \hat{C}^{2}=O\left(b^{2}\right), \hat{B}^{3} \hat{D}=O\left(b^{2}\right), \hat{B} \hat{C} \hat{D}=O\left(b^{3}\right)$ and $\hat{D}^{2}=O\left(b^{4}\right)$, we obtain

$$
\bar{\Delta}=\frac{\hat{B}^{2}}{27}\left\{4 \hat{B} \hat{D}-\hat{C}^{2}\right\}+O\left(b^{3}\right) .
$$

We now want to translate the inequalities on the coefficients given in (A.1) and (A.2) into inequalities concerning regions of the parameter space $P$ :

Let $\alpha_{1} \in\left(0, \frac{\beta_{1}}{1-\phi}\left\{1-\frac{\gamma_{2} \alpha_{3} \phi}{\beta_{3}}\right\}\right]$ or, in other words, $R_{1} \in\left(0, R_{1}^{a}\right]$. We then have, for $b>0$,

$$
1 \geq(1-\phi) R_{1}+\phi R_{3}=\frac{\alpha_{1}(1-\phi)}{\beta_{1}}+\frac{\gamma_{2} \alpha_{3} \phi}{\beta_{3}}>\frac{\alpha_{1}(1-\phi)}{b+\beta_{1}}+\frac{\gamma_{2} \alpha_{3} \phi}{b+\beta_{3}}=R_{0} .
$$

We firstly consider (A.1). Unless $R_{3}=1$ we certainly have that $\Delta>0$ for $b$ sufficiently small. At this point we introduce the notation $x \square y$ to represent the statement:

$$
\text { ' } x<y \text { for sufficiently small } b \text { ', }
$$

so the above becomes $\Delta \square 0$ unless $R_{3}=1$. Also, $R_{3}>1$ implies $\hat{B} \square 0$. Note that:

(1) For the above interval to exist we require that $R_{3}<1 / \phi$, if $\phi>0$.

(2) When $R_{1}$ is in this interval then $R_{3}>1$ implies that $R_{1}<1$.

Using the expression for $\hat{C}$ gives us that $R_{3}<\frac{1}{\phi}\left\{1-\frac{1-\phi}{1+\gamma_{1}}\left(\gamma_{1} R_{1}+R_{2}\right)\right\}$ implies that $\hat{C} \square 0$. For the above inequality to be compatible with $R_{3}>1$ we require that $\phi<1-\frac{1-\phi}{1+\gamma_{1}}\left(\gamma_{1} R_{1}+R_{2}\right)$ which gives, on rearranging, that $R_{2}<\gamma_{1}\left(1-R_{1}\right)+1$.

Since $R_{1}<1$ when $R_{3}>1, \gamma_{1}\left(1-R_{1}\right)+1>0$. We also require that $\bar{\Delta}<0$. We have 


$$
\begin{aligned}
\bar{\Delta}= & \frac{\hat{B}^{2}}{27}\left\{4 \hat{B} \hat{D}-\hat{C}^{2}\right\}+O\left(b^{3}\right) \\
= & \frac{\hat{B}^{2}}{27}\left\{4\left(\frac{\beta_{3}}{\gamma_{2}}\left(1-R_{3}\right)+O(b)\right)\left(\frac{b^{2} \beta_{3}}{\gamma_{1} \gamma_{2}} 1-(1-\phi) R_{1}-\phi R_{3}+O\left(b^{3}\right)\right)\right. \\
& \left.\quad-\left(\frac{b \beta_{3}}{\gamma_{1} \gamma_{2}}\left(1+\gamma_{1}\right)\left(1-\phi R_{3}\right)-(1-\phi)\left(\gamma_{1} R_{1}+R_{2}\right)+O\left(b^{2}\right)\right)^{2}\right\}+O\left(b^{3}\right) \\
= & \frac{\hat{B}^{2} b^{2} \beta_{3}^{2}}{27 \gamma_{1}^{2} \gamma_{2}^{2}} 4 \gamma_{1}\left(1-R_{3}\right) 1-(1-\phi) R_{1}-\phi R_{3} \\
& \quad-\left(1+\gamma_{1}\right)\left(1-\phi R_{3}\right)-(1-\phi)\left(\gamma_{1} R_{1}+R_{2}\right)^{2}+O\left(b^{3}\right) .
\end{aligned}
$$

Therefore if

$$
R_{3}>1, R_{1} \leq R_{1}^{a} \text { and } R_{3}<\frac{1}{\phi}\left\{1-\frac{1-\phi}{1+\gamma_{1}}\left(\gamma_{1} R_{1}+R_{2}\right)\right\}
$$

then $\bar{\Delta} \square 0$.

We now need to combine all the inequalities involving $R_{3}$. We have that the least upper bound imposed on $R_{3}$ by the condition $R_{1} \leq R_{1}^{a}$ is at most that imposed by the condition

$$
R_{3}<\frac{1}{\phi}\left\{1-\frac{1-\phi}{1+\gamma_{1}}\left(\gamma_{1} R_{1}+R_{2}\right)\right\}
$$

if, and only if, $R_{1} \geq R_{2}$, giving us that, for $R_{1} \in\left(0, R_{1}^{a}\right]$, (A.1) is satisfied, for sufficiently small $b$, when

or

$$
\begin{aligned}
& 1<R_{3}<\frac{1}{\phi}\left\{1-\frac{1-\phi}{1+\gamma_{1}}\left(\gamma_{1} R_{1}+R_{2}\right)\right\} \text { and } R_{1}<R_{2}<1+\gamma_{1}\left(1-R_{1}\right) \\
& 1<R_{3} \leq \frac{1}{\phi}\left\{1-(1-\phi) R_{1}\right\} \text { and } R_{2} \leq R_{1},
\end{aligned}
$$

noting that $1+\gamma_{1}\left(1-R_{1}\right)>1$ when $R_{1}<1$, as is the case when $R_{3}>1$, so that there always exists an $R_{2}$ satisfying the latter inequality in (A.3).

It is clearer to combine the above conditions to give that, when $R_{1} \in\left(0, R_{1}^{a}\right]$, (A.1) is satisfied, for sufficiently small $b$, when

$$
R_{2}<1+\gamma_{1}\left(1-R_{1}\right) \text { and } 1<R_{3}<\frac{1}{\phi}\left\{1-\frac{1-\phi}{1+\gamma_{1}} \max \gamma_{1} R_{1}+R_{2},\left(1+\gamma_{1}\right) R_{1}\right\}
$$

noting that the upper inequality on $R_{3}$ could be either $<$ or $\leq$, depending on which of $\gamma_{1} R_{1}+R_{2}$ or $\left(1+\gamma_{1}\right) R_{1}$ is actually the larger. The remaining conditions on $R_{2}$ in (A.3) and (A.4) will automatically be implied by the relative sizes of these expressions.

We now consider the set of inequalities on the coefficients in (A.2), again with $R_{1} \in\left(0, R_{1}^{a}\right]$. Once more we require that $R_{3}<1 / \phi$, and we also have that $\Delta \square 0$ 
unless $R_{3}=1 . \quad$ Next, $R_{3}>\frac{1}{\phi}\left\{1-\frac{1-\phi}{1+\gamma_{1}}\left(\gamma_{1} R_{1}+R_{2}\right)\right\}$ or the equivalent inequality, $R_{2}>\left(1+\gamma_{1}\right) R_{1}^{a}-\gamma_{1} R_{1}$, implies that $\hat{C} \square 0$.

As with (A.1) above we have that

$$
4 \gamma_{1}\left(1-R_{3}\right) 1-(1-\phi) R_{1}-\phi R_{3}<\left(1+\gamma_{1}\right)\left(1-\phi R_{3}\right)-(1-\phi)\left(\gamma_{1} R_{1}+R_{2}\right)^{2}
$$

implies that $\bar{\Delta} \square 0$. Thus if $R_{1} \leq R_{1}^{a}$ and $R_{3}>1$ then we have that $\bar{\Delta} \square 0$.

Finally, if $R_{1} \leq R_{1}^{a}$ and $R_{3}<1$ then, in order that $\bar{\Delta} \square 0$, we require either

$$
\begin{aligned}
& \left(1+\gamma_{1}\right)\left(1-\phi R_{3}\right)-(1-\phi)\left(\gamma_{1} R_{1}+R_{2}\right)>2 \sqrt{\gamma_{1}\left(1-R_{3}\right) 1-(1-\phi) R_{1}-\phi R_{3}} \\
& \text { or } \quad(1-\phi)\left(\gamma_{1} R_{1}+R_{2}\right)-\left(1+\gamma_{1}\right)\left(1-\phi R_{3}\right)>2 \sqrt{\gamma_{1}\left(1-R_{3}\right) 1-(1-\phi) R_{1}-\phi R_{3}} \text {. }
\end{aligned}
$$

(A.5) rearranges to

$$
R_{2}<\frac{1}{1-\phi}\left(1+\gamma_{1}\right)\left(1-\phi R_{3}\right)-2 \sqrt{\gamma_{1}\left(1-R_{3}\right) 1-(1-\phi) R_{1}-\phi R_{3}}-\gamma_{1} R_{1},
$$

which contradicts the requirement that $R_{2}>\left(1+\gamma_{1}\right) R_{1}^{a}-\gamma_{1} R_{1}$, while (A.6) rearranges to

$$
R_{2}>\frac{1}{1-\phi}\left(1+\gamma_{1}\right)\left(1-\phi R_{3}\right)+2 \sqrt{\gamma_{1}\left(1-R_{3}\right) 1-(1-\phi) R_{1}-\phi R_{3}}-\gamma_{1} R_{1}
$$

Summarising, for $R_{1} \in\left(0, R_{1}^{a}\right]$ with sufficiently small $b$, condition (A.2) is satisfied when either

$1<R_{3}<\frac{1}{\phi}$ and $R_{2}>\left(1+\gamma_{1}\right) R_{1}^{a}-\gamma_{1} R_{1}$

or $R_{3}<1$ and $\quad R_{2}>\frac{1}{1-\phi}\left(1+\gamma_{1}\right)\left(1-\phi R_{3}\right)+2 \sqrt{\gamma_{1}\left(1-R_{3}\right) 1-(1-\phi) R_{1}-\phi R_{3}}-\gamma_{1} R_{1}$.

We would now like to provide an overall summary, using $R_{1}$ as the bifurcation parameter, of the conditions on the parameter space appearing in (A.3), (A.4), (A.7) and (A.8). Thus we will need to obtain sets of inequalities, each of which is independent of $R_{1}$ and accompanied by an interval for which two subcritical endemic equilibria can exist when $R_{1}$ is contained in that interval.

We note here that, in order for two subcritical endemic equilibria to exist, it is enough that any one of these sets of conditions is satisfied. It is important to mention that, so long as we disregard the 'boundary sets' in $P$ such as $R_{3}=1$, a necessary and sufficient condition for a point in $P$ to allow the existence of two subcritical endemic equilibria is that it satisfies at least one of these sets of conditions.

We will show that the conditions in (A.3), (A.4) and (A.7) can be combined to give a particularly simple set of criteria for two subcritical endemic equilibria to exist in the case where $1<R_{3}<1 / \phi$. The conditions in (A.8) then cover the situation for 
which $R_{3}<1$. Since the situation is complicated, we build the picture up gradually. First we consider the conditions in each of (A.3), (A.4), (A.7) and (A.8) separately. Here we state only the conditions under which two subcritical endemic equilibria do exist, and it may therefore be assumed that, with the possible exception of a finite number of special case equalities defining lines, planes and surfaces in $P$ (that are on the boundaries of the regions we discuss, some of which will be highlighted later on) two subcritical endemic equilibria are not present for any set of conditions not covered in the following summary:

We have that, for sufficiently small $b$ :

From (A.3)

(1) If $1<R_{3}<\frac{1}{\phi}\left\{1-\frac{1-\phi}{1+\gamma_{1}} R_{2}\right\}$ and $R_{2}<1+\gamma_{2}$ then there are two subcritical endemic equilibria for all $R_{1} \in\left(0, \min \left\{R_{1}^{a}, R_{1}^{b}, R_{1}^{c}, R_{1}^{d}\right\}\right)$. It is straightforward to show that $\min \left\{R_{1}^{a}, R_{1}^{b}, R_{1}^{c}, R_{1}^{d}\right\}>0$. Note also that there exists an $R_{3}$ satisfying $1<R_{3}<\frac{1}{\phi}\left\{1-\frac{1-\phi}{1+\gamma_{1}} R_{2}\right\}$ if, and only if, $R_{2}<1+\gamma_{1}$.

From (A.4)

(2) If $1<R_{3}<\frac{1}{\phi}$ and $R_{2} \leq R_{1}^{a}$ then there are two endemic subcritical equilibria for $R_{1} \in\left[R_{1}^{c}, R_{1}^{a}\right]$, noting that $R_{1}^{c} \leq R_{1}^{a}$ if, and only if, $R_{2} \leq R_{1}^{a}$.

From (A.7)

(3) If $1<R_{3}<\frac{1}{\phi}$ and $R_{2} \geq\left(1+\gamma_{1}\right) R_{1}^{a}$ then there are two subcritical endemic equilibria for $R_{1} \in\left(0, R_{1}^{a}\right]$.

(4) If $1<R_{3}<\frac{1}{\phi}$ and $R_{1}^{a}<R_{2}<\left(1+\gamma_{1}\right) R_{1}^{a}$ then there are two subcritical endemic equilibria for $R_{1} \in\left(R_{1}^{b}, R_{1}^{a}\right]$. We also see that $R_{1}^{b}<R_{1}^{a}$ if, and only if, $R_{2}>R_{1}^{a}$, which is always satisfied in this case.

From (A.8)

(5) If $R_{3}<1$ and $R_{2} \geq \frac{1}{1-\phi}\left(1+\gamma_{1}\right)\left(1-\phi R_{3}\right)+2 \sqrt{\gamma_{1}\left(1-R_{3}\right) 1-\phi R_{3}}$ then there are two subcritical endemic equilibria for all $R_{1} \in\left(0, R_{1}^{a}\right]$.

(6) If $R_{3}<1$ and $R_{1}^{a}<R_{2}<\frac{1}{1-\phi}\left(1+\gamma_{1}\right)\left(1-\phi R_{3}\right)+2 \sqrt{\gamma_{1}\left(1-R_{3}\right) 1-\phi R_{3}}$ then there are two subcritical endemic equilibria for all $R_{1} \in\left(R_{1}^{e}, R_{1}^{a}\right]$.

Before attempting to combine the above inequalities, we consider the special case $R_{1}=R_{1}^{b}$, which will be useful in due course. We have that

$$
\bar{\Delta}=\frac{4 \hat{B}^{2} b^{2} \beta_{3}^{2}}{27 \gamma_{1} \gamma_{2}^{2}}\left(1-R_{3}\right)\left(1-(1-\phi) R_{1}-\phi R_{3}\right)+O\left(b^{3}\right)
$$


from which we see that if $R_{3}>1$ then $\bar{\Delta} \square 0$ provided that $R_{1}<R_{1}^{a}$, i.e. provided that $R_{1} \in\left(0, R_{1}^{a}\right)$ rather than $R_{1} \in\left(0, R_{1}^{a}\right]$. Thus (A.1) will be satisfied when $\hat{C} \geq 0$, bearing in mind that $\Delta \square 0$ and $\hat{B} \square 0$ whenever $R_{3}>1$. However, if $R_{3}>1$ and $\hat{C}<0$ then we see that (A.2) will be satisfied. Thus two subcritical endemic equilibria exist when $0<R_{1}=R_{1}^{b}<R_{1}^{a}$ and $R_{3}>1$.

Now we combine (1) and (2) to give us that if $1<R_{3}<1 / \phi$ and $R_{2} \leq R_{1}^{a}$ then there are two subcritical endemic equilibria for all $R_{1} \in\left(0, \min \left\{R_{1}^{a}, R_{1}^{b}, R_{1}^{c}, R_{1}^{d}\right\}\right) \cup\left[R_{1}^{c}, R_{1}^{a}\right]$. Under the above conditions we have that $\min \left\{R_{1}^{a}, R_{1}^{b}, R_{1}^{c}, R_{1}^{d}\right\}=R_{1}^{c} \quad$ (as easily checked) so that there are two subcritical endemic equilibria for all $R_{1} \in\left(0, R_{1}^{a}\right]$.

Next we combine (1) and (4) to obtain the result that if

$$
1<R_{3}<\frac{1}{\phi} \text { and } R_{1}^{a}<R_{2}<\left(1+\gamma_{1}\right) R_{1}^{a}
$$

then there are two subcritical endemic equilibria for all $R_{1} \in\left(0, \min \left\{R_{1}^{a}, R_{1}^{b}, R_{1}^{c}, R_{1}^{d}\right\}\right) \cup\left(R_{1}^{b}, R_{1}^{a}\right]$. Under the above conditions, $\min \left\{R_{1}^{a}, R_{1}^{b}, R_{1}^{c}, R_{1}^{d}\right\}=R_{1}^{b}$ so that there are two subcritical endemic equilibria for all $R_{1} \in\left(0, R_{1}^{b}\right) \cup\left(R_{1}^{b}, R_{1}^{a}\right]$. Using the result we obtained for the special case $R_{1}=R_{1}^{b}$ (in the paragraph immediately after (6)), we have that, under the above conditions, there are two subcritical endemic equilibria for all $R_{1} \in\left(0, R_{1}^{a}\right)$.

Now (3), in conjunction with the results from the previous two paragraphs, tells us that, for sufficiently small $b$, two subcritical endemic equilibria can occur when $R_{1}<R_{1}^{a}$ and $1<R_{3}<1 / \phi$.

Via some further analysis we may extend the conditions to cover the case $R_{3}=1$. Details are given in Griffiths (2007). Then, with the results from (5) and (6), we deduce that Theorem 4.1 is true.

Proof of Theorem 4.2 As $R_{0}>1$ we have that $\hat{D}<0$ so that $F(I)=0$ possesses either one or three positive roots (i.e. there will either be one or three supercritical endemic equilibria). Once more, by considering the possible shapes of the graph $y=F(I)$ we obtain: If $R_{0}>1$ there are three endemic equilibria if, and only if,

$$
\Delta>0, \hat{B}<0, \hat{C}>0 \text { and } \bar{\Delta}<0 .
$$

The above conditions are the same as those in (A.1) for the subcritical case, other than the fact that we cannot now have $\hat{C}=0$. Now, however, we have that $\hat{D}<0$ and $R_{0}=\frac{\alpha_{1}(1-\phi)}{b+\beta_{1}}+\frac{\gamma_{2} \alpha_{3} \phi}{b+\beta_{3}}>1$. The latter implies that

$$
(1-\phi) R_{1}+\phi R_{3}=\frac{\alpha_{1}(1-\phi)}{\beta_{1}}+\frac{\gamma_{2} \alpha_{3} \phi}{\beta_{3}}>1
$$


and hence that $R_{1}>R_{1}^{a}$. However, we also note here that

$$
\frac{\alpha_{1}(1-\phi)}{\beta_{1}}+\frac{\gamma_{2} \alpha_{3} \phi}{\beta_{3}}>1 \text { implies that } R_{0}=\frac{\alpha_{1}(1-\phi)}{b+\beta_{1}}+\frac{\gamma_{2} \alpha_{3} \phi}{b+\beta_{3}}>1
$$

for sufficiently small $b$.

Once more we have that $R_{3}>1$ implies that $\hat{B} \square 0$ and $\Delta \square 0$. Also,

or equivalently,

$$
R_{3}<\frac{1}{\phi}\left\{1-\frac{1-\phi}{1+\gamma_{1}}\left(\gamma_{1} R_{1}+R_{2}\right)\right\}
$$

implies that $\hat{C} \square 0$, but, for

$$
R_{2}<\left(1+\gamma_{1}\right) R_{1}^{a}-\gamma_{1} R_{1}
$$

$$
R_{3}>1 \text { and } R_{3}<\frac{1}{\phi}\left\{1-\frac{1-\phi}{1+\gamma_{1}}\left(\gamma_{1} R_{1}+R_{2}\right)\right\}
$$

to be compatible we require that

$$
R_{2}<\gamma_{1}\left(1-R_{1}\right)+1 \text {. }
$$

This provides us with the restriction $R_{1}<1+1 / \gamma_{1}$. For $R_{3}>1$ this is compatible with $R_{1}>R_{1}^{a}$.

However, since $\hat{D}<0$ and we require $\hat{B}<0$ for there to be any possibility of three endemic equilibria occuring, then $\hat{B} \hat{D}$ must be positive and we cannot therefore assume now that $\bar{\Delta} \square 0$ as we did in case (A.1) for when $R_{0}<1$. This time we require that

$$
\begin{aligned}
\bar{\Delta} & =\frac{\hat{B}^{2} b^{2} \beta_{3}^{2}}{27 \gamma_{1}^{2} \gamma_{2}^{2}} 4 \gamma_{1}\left(1-R_{3}\right) 1-(1-\phi) R_{1}-\phi R_{3} \\
& <0 \\
& -\left(1+\gamma_{1}\right)\left(1-\phi R_{3}\right)-(1-\phi)\left(\gamma_{1} R_{1}+R_{2}\right)^{2}+O\left(b^{3}\right)
\end{aligned}
$$

subject to the conditions $R_{1}>R_{1}^{a}$ and $R_{3}>1$. In this case we have that

$$
\left(1-R_{3}\right) 1-(1-\phi) R_{1}-\phi R_{3}>0
$$

so, in order that $\bar{\Delta} \square 0$ we require, as in case (A.2) for $R_{0}<1$, that

$$
\begin{array}{r}
\left(1+\gamma_{1}\right)\left(1-\phi R_{3}\right)-(1-\phi)\left(\gamma_{1} R_{1}+R_{2}\right)>2 \sqrt{\gamma_{1}\left(R_{3}-1\right)(1-\phi) R_{1}+\phi R_{3}-1} \\
\text { or } \quad(1-\phi)\left(\gamma_{1} R_{1}+R_{2}\right)-\left(1+\gamma_{1}\right)\left(1-\phi R_{3}\right)>2 \sqrt{\gamma_{1}\left(R_{3}-1\right)(1-\phi) R_{1}+\phi R_{3}-1} .
\end{array}
$$

(A.12) rearranges to

$$
R_{2}<\frac{1}{1-\phi}\left(1+\gamma_{1}\right)\left(1-\phi R_{3}\right)-2 \sqrt{\gamma_{1}\left(R_{3}-1\right)(1-\phi) R_{1}+\phi R_{3}-1}-\gamma_{1} R_{1},
$$


while (A.13) is incompatible with (A.10).

Now, since $R_{2}$ must be positive, we require from (A.12) that

$$
\frac{1}{1-\phi}\left(1+\gamma_{1}\right)\left(1-\phi R_{3}\right)-2 \sqrt{\gamma_{1}\left(R_{3}-1\right)(1-\phi) R_{1}+\phi R_{3}-1}-\gamma_{1} R_{1}>0 .
$$

If we write $x=\gamma_{1}\left(1-(1-\phi) R_{1}-\phi R_{3}\right)$ then the quadratic inequality in $x$ given by

$$
\left(x+1-\phi R_{3}\right)^{2}>-4 x\left(R_{3}-1\right)
$$

will furnish us with solutions to (A.15) provided that $x+1-\phi R_{3}>0$, which, as is easily checked, is equivalent to $\left(1+\gamma_{1}\right) R_{1}^{a}-\gamma_{1} R_{1}>0$. However using (A.10) this must be true.

(A.16) rearranges to

$$
x^{2}+2(2-\phi) R_{3}-1 x+\left(1-\phi R_{3}\right)^{2}>0
$$

and is satisfied when either $x<-c-\sqrt{c^{2}-d^{2}}$ or $x>-c+\sqrt{c^{2}-d^{2}}$, where $c=(2-\phi) R_{3}-1$ and $d=1-\phi R_{3}$. We have that

$$
c^{2}-d^{2}=4(1-\phi) R_{3}\left(R_{3}-1\right)
$$

and the above inequalities then give us that

$$
\begin{array}{ll} 
& \gamma_{1}(1-\phi) R_{1}>\gamma_{1}\left(1-\phi R_{3}\right)+(2-\phi) R_{3}-1+2 \sqrt{(1-\phi) R_{3}\left(R_{3}-1\right)} \\
\text { or } \quad & \gamma_{1}(1-\phi) R_{1}<\gamma_{1}\left(1-\phi R_{3}\right)+(2-\phi) R_{3}-1-2 \sqrt{(1-\phi) R_{3}\left(R_{3}-1\right)} .
\end{array}
$$

(A.17) implies that

$$
\gamma_{1}(1-\phi) R_{1}>\gamma_{1}\left(1-\phi R_{3}\right)+\left(2 R_{3}-1\right)-\phi R_{3},
$$

which gives us, since $R_{3}>1$, that

$$
\gamma_{1}(1-\phi) R_{1}>\gamma_{1}\left(1-\phi R_{3}\right)+1-\phi R_{3}=\left(1+\gamma_{1}\right)\left(1-\phi R_{3}\right) .
$$

This is incompatible with (A.10).

For (A.18) we have that $(2-\phi) R_{3}-1^{2}-4(1-\phi) R_{3}\left(R_{3}-1\right)=\left(\phi R_{3}-1\right)^{2}>0$, so that $(2-\phi) R_{3}-1>2 \sqrt{(1-\phi) R_{3}\left(R_{3}-1\right)}$, noting that $(2-\phi) R_{3}-1$ is certainly positive since $R_{3}>1$ and $0<\phi<1$. We therefore see that (A.18) is compatible with $R_{1}>R_{1}^{a}$.

Before summarising the conditions under which there exist three supercritical endemic equilibria we need to compare the following three requirements on $R_{2}$ : (A.10), (A.11) and (A.14). We have that $R_{3}>1$ implies that $\left(1+\gamma_{1}\right) R_{1}^{a}-\gamma_{1} R_{1}<\gamma_{1}\left(1-R_{1}\right)+1$. Also, the right hand side of (A.14) is smaller than the right hand of (A.10) so it is (A.14) that we need. 
We now have the result that, for $R_{0}>1$ and $b$ sufficiently small, three endemic equilibria occur when

$$
\begin{array}{ll} 
& \frac{1-\phi R_{3}}{1-\phi}<R_{1}<\frac{1}{1-\phi}\left(1-\phi R_{3}+\frac{1}{\gamma_{1}}(2-\phi) R_{3}-1-2 \sqrt{(1-\phi) R_{3}\left(R_{3}-1\right)}\right), \\
& R_{2}<\frac{1}{1-\phi}\left(1+\gamma_{1}\right)\left(1-\phi R_{3}\right)-2 \sqrt{\gamma_{1}\left(R_{3}-1\right)(1-\phi) R_{1}+\phi R_{3}-1}-\gamma_{1} R_{1} \\
\text { and } \quad 1<R_{3}<\frac{1}{\phi}\left\{1-\frac{1-\phi}{1+\gamma_{1}}\left(\gamma_{1} R_{1}+R_{2}\right)\right\} .
\end{array}
$$

These inequalities are not in a particularly pleasant form as they stand, so we now unravel them somewhat. Let us define

$$
R_{2}^{u}\left(R_{1}\right)=\frac{1}{1-\phi}\left(1+\gamma_{1}\right)\left(1-\phi R_{3}\right)-2 \sqrt{\gamma_{1}\left(R_{3}-1\right)(1-\phi) R_{1}+\phi R_{3}-1}-\gamma_{1} R_{1} .
$$

Now, for $R_{3}>1, R_{2}^{u}\left(R_{1}\right)$ is a decreasing function on $\left(R_{1}^{a}, R_{1}^{t}\right)$ with $R_{2}^{u}\left(R_{1}^{a}\right)=R_{1}^{a}$ and $R_{2}^{u}\left(R_{1}^{t}\right)=0$. In order to show that $R_{2}^{u}\left(R_{1}^{t}\right)=0$ we note that

$$
(1-\phi) R_{1}^{t}+\phi R_{3}-1=\frac{1}{\gamma_{1}} \sqrt{(1-\phi) R_{3}}-{\sqrt{R_{3}-1}}^{2},
$$

and that $R_{3}<1 / \phi$ implies that $(1-\phi) R_{3}>R_{3}-1$ so we have

$$
\sqrt{(1-\phi) R_{1}^{t}+\phi R_{3}-1}=\frac{1}{\sqrt{\gamma_{1}}} \sqrt{(1-\phi) R_{3}}-\sqrt{R_{3}-1},
$$

giving us, after some simplification,

$$
\begin{aligned}
(1-\phi) R_{2}^{u}\left(R_{1}^{t}\right) & =\left(1-\phi R_{3}\right)-\sqrt{(1-\phi) R_{3}}-{\sqrt{R_{3}-1}}^{2}-2 \sqrt{(1-\phi) R_{3}}-\sqrt{R_{3}-1} \sqrt{R_{3}-1} \\
& =0
\end{aligned}
$$

as required.

Next, note that $R_{1}^{a}$ and 0 are greatest lower bounds for $R_{1}$ and $R_{2}$ respectively (in the situation we are currently considering), so that, as is easily checked, three supercritical endemic equilibria are possible for any $R_{3}$ such that $1<R_{3}<1 / \phi$.

We also note that since $R_{2}^{u}\left(R_{1}^{t}\right)=0$ the inequality

$$
R_{2} \leq \frac{1}{1-\phi}\left(1+\gamma_{1}\right)\left(1-\phi R_{3}\right)-2 \sqrt{\gamma_{1}\left(R_{3}-1\right)(1-\phi) R_{1}+\phi R_{3}-1}-\gamma_{1} R_{1}
$$

cannot be satisfied for any positive values of $R_{2}$ when $R_{1}=R_{1}^{t}$. We are assuming that all parameters are positive so this allows us to state in (2) of Theorem 4.2 that there is only one supercritical endemic equilibrium for all $R_{1} \in\left(R_{1}^{*}, \infty\right)$, rather than just for all $R_{1} \in\left(R_{1}^{*}, R_{1}^{t}\right) \cup\left(R_{1}^{t}, \infty\right)$. This completes the proof of Theorem 4.2. 